ESAIM: M2AN 46 (2012) 661-680

DOI: $10.1051 / \mathrm{m} 2 \mathrm{an} / 2011059$
ESAIM: Mathematical Modelling and Numerical Analysis

www.esaim-m2an.org

\title{
CONSTRAINT PRESERVING SCHEMES USING POTENTIAL-BASED FLUXES. III. GENUINELY MULTI-DIMENSIONAL SCHEMES FOR MHD EQUATIONS *,**
}

\author{
SidDhartha Mishra ${ }^{1}$ AND Eitan TADMOR ${ }^{2}$
}

\begin{abstract}
We design efficient numerical schemes for approximating the MHD equations in multidimensions. Numerical approximations must be able to deal with the complex wave structure of the MHD equations and the divergence constraint. We propose schemes based on the genuinely multidimensional (GMD) framework of [S. Mishra and E. Tadmor, Commun. Comput. Phys. 9 (2010) 688-710; S. Mishra and E. Tadmor, SIAM J. Numer. Anal. 49 (2011) 1023-1045]. The schemes are formulated in terms of vertex-centered potentials. A suitable choice of the potential results in GMD schemes that preserve a discrete version of divergence. First- and second-order divergence preserving GMD schemes are tested on a series of benchmark numerical experiments. They demonstrate the computational efficiency and robustness of the GMD schemes.
\end{abstract}

Mathematics Subject Classification. 65M06, 35L65.

Received September 27, 2009. Revised May 23, 2010.

Published online January 11, 2012.

\section{INTRODUCTION}

Modeling of plasmas lies at the core of many interesting problems in astrophysics, solar physics, electrical and aerospace engineering. Macroscopic plasma dynamics is characterized by the interaction of the moving plasma with the magnetic field which is often modeled by the equations of ideal Magnetohydrodynamics (MHD). In

Keywords and phrases. Multidimensional evolution equations, magnetohydrodynamics, constraint transport, central difference schemes, potential-based fluxes.

* To the memory of David Gottlieb.

** The work on this paper was started when S.M. visited the Center of Scientific Computation and Mathematical Modeling (CSCAMM) and he thanks CSCAMM and all its members for the excellent hospitality and facilities. E. T. Research was supported in part by NSF grants DMS07-07949, DMS10-08397 and ONR grant N00014-091-0385. He thanks the Centre for Advanced Study at the Norwegian Academy of Science and Letters, for hosting him as part of its international research program on Nonlinear PDEs during the academic year 2008-09.

1 Centre of Mathematics for Applications (CMA), University of Oslo, P.O. Box 1053, Blindern, 0316 Oslo, Norway. siddharm@cma.uio.no.

2 Department of Mathematics, Center of Scientific Computation and Mathematical Modeling (CSCAMM), Institute for Physical sciences and Technology (IPST), University of Maryland, 20741-4015 MD, Maryland, USA. tadmor@cscamm.umd.edu 
two space dimensions, the MHD equations are

$$
\mathbf{U}_{t}+\mathbf{f}(\mathbf{U})_{x}+\mathbf{g}(\mathbf{U})_{y}=0, \quad(x, y, t) \in \mathbb{R} \times \mathbb{R} \times \mathbb{R}_{+},
$$

where

$$
\mathbf{U}=\left(\begin{array}{l}
\rho \\
\rho u_{1} \\
\rho u_{2} \\
\rho u_{3} \\
B_{1} \\
B_{2} \\
B_{3} \\
E
\end{array}\right), \mathbf{f}=\left(\begin{array}{l}
\rho u_{1} \\
\rho\left(u_{1}\right)^{2}+\widetilde{p}-\frac{1}{2}\left(B_{1}\right)^{2} \\
\rho u_{1} u_{2}-B_{1} B_{2} \\
\rho u_{1} u_{3}-B_{1} B_{3} \\
0 \\
-\left(u_{2} B_{1}-u_{1} B_{2}\right) \\
u_{1} B_{3}-u_{3} B_{1} \\
(E+\widetilde{p}) u_{1}-(\mathbf{u} \cdot \mathbf{B}) B_{1}
\end{array}\right), \mathbf{g}=\left(\begin{array}{l}
\rho u_{2} \\
\rho u_{1} u_{2}-B_{1} B_{2} \\
\rho\left(u_{2}\right)^{2}+\widetilde{p}-\frac{1}{2}\left(B_{2}\right)^{2} \\
\rho u_{1} u_{3}-B_{1} B_{3} \\
u_{2} B_{1}-u_{1} B_{2} \\
0 \\
u_{2} B_{3}-u_{3} B_{2} \\
(E+\widetilde{p}) u_{2}-(\mathbf{u} \cdot \mathbf{B}) B_{2}
\end{array}\right)
$$

Here, $\rho$ denotes the density of the plasma and $\mathbf{u}=\left(u_{1}, u_{2}, u_{3}\right)^{\top}, \mathbf{B}=\left(B_{1}, B_{2}, B_{3}\right)^{\top}$ are, respectively, the velocity and magnetic fields, $E$ is the total energy and $\widetilde{p}$ is the total pressure,

$$
\widetilde{p}=p+\frac{1}{2}|\mathbf{B}|^{2},
$$

where $p$ is the thermal pressure dictated by the equation of state of an ideal gas with a gas constant $\gamma$,

$$
E=\frac{p}{\gamma-1}+\frac{1}{2}\left(\rho|\mathbf{u}|^{2}+|\mathbf{B}|^{2}\right)
$$

The MHD equations combine conservation laws of the mass, momentum and energy with the magnetic induction equations. The special structure of the equations in their two-dimensional setup (1.1) is observed in the 8-vector fluxes which satisfy

$$
\mathbf{f}_{5}=\mathbf{g}_{6} \equiv 0, \quad-\mathbf{f}_{6}=\mathbf{g}_{5}=h, \quad h \equiv h(\mathbf{U}):=u_{2} B_{1}-u_{1} B_{2} .
$$

For an arbitrary 8 -vector $\mathbf{w}$, we let $\widetilde{\mathbf{w}}$ denote the reduced 6 -vector $\widetilde{\mathbf{w}}:=\left(w_{1}, w_{2}, w_{3}, w_{4}, w_{7}, w_{8}\right)^{\top}$; then we can rewrite (1.1), (1.2) in the equivalent form

$$
\begin{aligned}
\widetilde{\mathbf{U}}_{t}+\widetilde{\mathbf{f}}(\mathbf{U})_{x}+\widetilde{\mathbf{g}}(\mathbf{U})_{y} & =0, \\
\left(B_{1}\right)_{t}+h(\mathbf{U})_{y} & =0, \\
\left(B_{2}\right)_{t}-h(\mathbf{U})_{x} & =0 .
\end{aligned}
$$

The last two equations imply that solutions of (1.1) satisfy the two-dimensional divergence constraint

$$
\left(\operatorname{div}\left(\left(B_{1}, B_{2}\right)^{\top}\right)\right)_{t} \equiv 0 .
$$

Similarly, the magnetic field in the MHD equations in their three-dimensional setup satisfy the special form of the Maxwell's equations, e.g., [36],

$$
\mathbf{B}_{t}+\operatorname{curl}(\mathbf{B} \times \mathbf{u})=0, \quad(x, y, t) \in \mathbb{R} \times \mathbb{R} \times \mathbb{R}_{+},
$$

which in turn, implies the divergence constraint:

$$
(\operatorname{div} \mathbf{B})_{t} \equiv 0
$$

Since magnetic monopoles have not been observed in nature, the initial magnetic field is assumed to be divergence free. The divergence constraints (1.4), (1.6) imply that the divergence of the magnetic field remains zero.

The system of ideal MHD equations is an example for a non-strictly hyperbolic system of conservation laws with an intrinsic constraint [37]. The solutions of a non-linear system like (1.1) develop generic discontinuities in the form of shock waves and contact discontinuities. The lack of strict hyperbolicity and the non-convexity of the MHD equations lead to a complex shock structure, consisting of intermediate and compound shocks [38]. Consequently, relatively few theoretical results are currently available for the MHD equations and numerical methods for the approximate solution of these equations are sought. 


\subsection{Finite-volume schemes}

Finite-volume methods are among the most widely used numerical methods for the approximate solution of systems of conservation laws such as the MHD equations (1.1), see [25,41] and the references therein. In a finite volume approximation, the computational domain is discretized into cells and an integral form of the conservation law (1.1) is realized on each cell in terms of cell averages. To this end we cover the $x-y$ plane with discrete cells, $\mathcal{C}_{i, j}:=\left[x_{i-\frac{1}{2}}, x_{i+\frac{1}{2}}\right) \times\left[y_{j-\frac{1}{2}}, y_{j+\frac{1}{2}}\right)$, centered at the mesh points $\left(x_{i}, y_{j}\right)=(i \Delta x, j \Delta y),(i, j) \in \mathbb{Z}^{2}$ with fixed mesh sizes $\Delta x, \Delta y$ in the $x$ - and $y$-directions. The cell averages of $\mathbf{U}$ over $\mathcal{C}_{i, j}$ at time $t$, denoted $\mathbf{U}_{i, j}(t)$, are then evolved by the semi-discrete scheme [25,41]:

$$
\frac{\mathrm{d}}{\mathrm{d} t} \mathbf{U}_{\mathbf{i}, \mathbf{j}}=-\frac{1}{\Delta x}\left(\mathbf{F}_{i+\frac{1}{2}, j}-\mathbf{F}_{i-\frac{1}{2}, j}\right)-\frac{1}{\Delta y}\left(\mathbf{G}_{i, j+\frac{1}{2}}-\mathbf{G}_{i, j-\frac{1}{2}}\right) .
$$

The time dependence of all the quantities in the above expression is suppressed for notational convenience. Classical first-order schemes employ two-point numerical fluxes of the form

$$
\mathbf{F}_{i+\frac{1}{2}, j}=\mathbf{F}\left(\mathbf{U}_{i, j}, \mathbf{U}_{i+1, j}\right), \quad \mathbf{G}_{i, j+\frac{1}{2}}=\mathbf{G}\left(\mathbf{U}_{i, j}, \mathbf{U}_{i, j+1}\right) .
$$

A canonical example is provided by the first-order Rusanov numerical flux:

$$
\begin{aligned}
\mathbf{F}_{i+\frac{1}{2}, j} & =\frac{1}{2}\left(\mathbf{f}\left(\mathbf{U}_{i, j}\right)+\mathbf{f}\left(\mathbf{U}_{i+1, j}\right)\right)-\max \left\{\left|\alpha_{i, j}\right|,\left|\alpha_{i+1, j}\right|\right\} \mathbf{J}\left(\mathbf{U}_{i+1, j}-\mathbf{U}_{i, j}\right), \\
\mathbf{G}_{i, j+\frac{1}{2}} & =\frac{1}{2}\left(\mathbf{g}\left(\mathbf{U}_{i, j}\right)+\mathbf{g}\left(\mathbf{U}_{i, j+1}\right)\right)-\max \left\{\left|\beta_{i, j}\right|,\left|\beta_{i, j+1}\right|\right\}\left(\mathbf{U}_{i, j+1}-\mathbf{U}_{i, j}\right) .
\end{aligned}
$$

Here, $\mathbf{J}$ is the $8 \times 8$ scaling matrix:

$$
\mathbf{J}=\operatorname{diag}\{1,1,1,1,1,-1,1,1\},
$$

reflecting the special structure in the opposite signs, $\mathbf{f}_{5}=-\mathbf{g}_{6}$, reflected in the fluxes specified in $(1.3 \mathrm{~b})$ and (1.3c). The $\alpha_{i, j}$ and $\beta_{i, j}$ in (1.9) are the maximal eigenvalues of the corresponding Jacobians $\mathbf{A}:=\partial_{\mathbf{U}} \mathbf{f}$ and $\mathbf{B}:=\partial_{\mathbf{U}} \mathbf{g}$ at a given state $\mathbf{U}_{i, j}$, i.e.,

$$
\alpha_{i, j}=\left|\left(u_{1}\right)_{i, j}\right|+\left|c_{1}\right|, \quad \beta_{i, j}=\left|\left(u_{2}\right)_{i, j}\right|+\left|c_{2}\right| ;
$$

here

$$
c_{k}^{2}=\frac{1}{2}\left(a_{i, j}^{2}+\left|\mathbf{b}_{i, j}\right|^{2}+\sqrt{\left(a_{i, j}^{2}+\left|\mathbf{b}_{i, j}\right|^{2}\right)^{2}-4 a_{i, j}^{2} b_{k}^{2}}\right), \quad k=1,2,
$$

where

$$
a_{i, j}^{2}:=\frac{\gamma p_{i, j}}{\rho_{i, j}}, \quad \text { and }\left|\mathbf{b}_{i, j}\right|^{2}=\left(b_{1}\right)_{i, j}^{2}+\left(b_{2}\right)_{i, j}^{2}+\left(b_{3}\right)_{i, j}^{2}, \quad \mathbf{b}_{i, j}:=\frac{\mathbf{B}_{i, j}}{\sqrt{\rho_{i, j}}} .
$$

The Rusanov flux (1.9) has been used in [2,3] as a simple yet highly effective building block for solving the ideal MHD equations using high-resolution central schemes. Other popular numerical fluxes used for the solution of MHD equations include linearized Roe solvers [9,36,37] and HLL type solvers [7, 17, 21,26,32]. Detailed comparisons of different solvers are performed in $[18,31]$. The Rusanov flux is particularly simple since it is free of the eigenstructure of the Jacobians - only a local estimate on the wave speeds is needed. But the resolution of these various fluxes is limited by their first-order accuracy.

The first-order accuracy of the two-point schemes (1.7), (1.8) can be extended to higher order by employing numerical fluxes which are based on wider, $2 p$-point stencils, $I_{i+\frac{1}{2}}:=\left\{i^{\prime}|| i^{\prime}-i-1 / 2 \mid<p\right\}$ along the $x$-axis and $J_{j+\frac{1}{2}}:=\left\{j^{\prime}|| j^{\prime}-j-1 / 2 \mid<p\right\}$ along the $y$-axis,

$$
\mathbf{F}_{i+\frac{1}{2}, j}=\mathbf{F}\left(\left\{\mathbf{U}_{i^{\prime}, j}\right\}_{i^{\prime} \in I_{i+\frac{1}{2}}}\right), \quad \mathbf{G}_{i, j+\frac{1}{2}}=\mathbf{G}\left(\left\{\mathbf{U}_{i, j^{\prime}}\right\}_{j^{\prime} \in J_{j+\frac{1}{2}}}\right) .
$$


The building blocks for such extensions are still the two-point numerical fluxes, $\mathbf{F}(\cdot, \cdot)$ and $\mathbf{G}(\cdot, \cdot)$. As a prototype example, we recall the class of second-order schemes based on piecewise bilinear MUSCL reconstruction [45],

$$
\mathbf{p}_{i, j}(x, y):=\mathbf{U}_{i, j}+\frac{\mathbf{U}_{i, j}^{\prime}}{\Delta x}\left(x-x_{i}\right)+\frac{\mathbf{U}_{i, j}^{\prime}}{\Delta y}\left(y-y_{j}\right) .
$$

Here, $\mathbf{U}^{\prime}$ and $\mathbf{U}^{\prime}$ denote the numerical derivatives

$$
\begin{aligned}
& \mathbf{U}_{i, j}^{\prime}=\operatorname{minmod}\left(\mathbf{U}_{i+1, j}-\mathbf{U}_{i, j}, \frac{1}{2}\left(\mathbf{U}_{i+1, j}-\mathbf{U}_{i-1, j}\right), \mathbf{U}_{i, j}-\mathbf{U}_{i-1, j}\right), \\
& \mathbf{U}_{i, j}^{\prime}=\operatorname{minmod}\left(\mathbf{U}_{i, j+1}-\mathbf{U}_{i, j}, \frac{1}{2}\left(\mathbf{U}_{i, j+1}-\mathbf{U}_{i, j-1}\right), \mathbf{U}_{i, j}-\mathbf{U}_{i, j-1}\right),
\end{aligned}
$$

which utilize the minmod limiter

$$
\operatorname{minmod}(a, b, c):= \begin{cases}\operatorname{sgn}(a) \min \{|a|,|b|,|c|\}, & \text { if } \operatorname{sgn}(a)=\operatorname{sgn}(b)=\operatorname{sgn}(c), \\ 0, & \text { otherwise }\end{cases}
$$

In this manner, one can reconstruct in each cell $\mathcal{C}_{i, j}$, the point values

$$
\mathbf{U}_{i, j}^{E}:=\mathbf{p}_{i, j}\left(x_{i+\frac{1}{2}}, y_{j}\right), \quad \mathbf{U}_{i, j}^{W}:=\mathbf{p}_{i, j}\left(x_{i-\frac{1}{2}}, y_{j}\right), \quad \mathbf{U}_{i, j}^{N}:=\mathbf{p}_{i, j}\left(x_{i}, y_{j+\frac{1}{2}}\right), \quad \mathbf{U}_{i, j}^{S}:=\mathbf{p}_{i, j}\left(x_{i}, y_{j-\frac{1}{2}}\right),
$$

from the given neighboring cell averages $\mathbf{U}_{i, j}, \mathbf{U}_{i \pm 1, j}$ and $\mathbf{U}_{i, j}, \mathbf{U}_{i, j \pm 1}$. The resulting second-order fluxes are then given by

$$
\mathbf{F}_{i+\frac{1}{2}, j}=\mathbf{F}\left(\mathbf{U}_{i, j}^{E}, \mathbf{U}_{i+1, j}^{W}\right), \quad \mathbf{G}_{i, j+\frac{1}{2}}=\mathbf{G}\left(\mathbf{U}_{i, j}^{N}, \mathbf{U}_{i, j+1}^{S}\right)
$$

The use of minmod limiter ensures the non-oscillatory behavior of the second-order schemes (1.7), (1.12). Observe that the resulting second-order MUSCL fluxes in (1.12e) are based on 4-point stencils

$$
\mathbf{F}_{i+\frac{1}{2}, j}=\mathbf{F}\left(\mathbf{U}_{i-1, j}, \mathbf{U}_{i, j}, \mathbf{U}_{i+1, j}, \mathbf{U}_{i+2, j}\right), \quad \mathbf{G}_{i, j+\frac{1}{2}}=\mathbf{G}\left(\mathbf{U}_{i, j-1}, \mathbf{U}_{i, j}, \mathbf{U}_{i, j+1}, \mathbf{U}_{i, j+2}\right) .
$$

Similar reconstructions together with upwind or central averaging yield a large class of high-resolution finitevolume semi-discrete schemes, e.g., $[22,23,33,40]$, which could then be integrated in time using standard stable high order Runge-Kutta methods [20].

\subsection{Genuinely multi-dimensional (GMD) fluxes}

Despite their considerable success, finite volume schemes (1.7) are known to be deficient in resolving genuinely multi-dimensional (GMD) waves [25]. Observe that the numerical fluxes $\mathbf{F}_{i+\frac{1}{2}, j}, \mathbf{G}_{i, j+\frac{1}{2}}$ in (1.11) are based on one-dimensional stencils which are supported in each normal direction but lack explicit transverse information. This could result in poor approximation of genuinely multi-dimensional waves, particularly for complicated systems like the ideal MHD equations (1.1). A characteristic feature of the MHD equations in this context, are the divergence constraints (1.4), (1.6), which reflect the essential multi-dimensional character of the MHD equations. Considerable effort has been devoted for devising numerical methods which address the multi-dimensional character of nonlinear system such as (1.1). These methods include dimensional splitting [25], wave propagation algorithms [24,25], method of transport [15,16,34], bi-characteristics based evolution Galerkin methods [27,28] and fluctuation splitting schemes [12].

The absence of an optimal strategy for designing GMD schemes for constrained systems of conservation laws such as the MHD equations, leaves room for designing stable GMD schemes that are easy to formulate and code, have a low computational cost and preserve other desirable properties rendered by the multi-dimensional structure of the system (1.1) like the divergence constraint. Their numerical fluxes take a general form

$$
\mathbf{F}_{i+\frac{1}{2}, j}=\mathbf{F}\left(\left\{\mathbf{U}_{\left(i^{\prime}, j^{\prime}\right) \in S_{i+\frac{1}{2}, j}}\right\}\right), \quad \mathbf{G}_{i, j+\frac{1}{2}}=\mathbf{G}\left(\left\{\mathbf{U}_{\left(i^{\prime}, j^{\prime}\right) \in S_{i, j+\frac{1}{2}}}\right\}\right) .
$$


Here, $S_{i+\frac{1}{2}, j}$ and $S_{i, j+\frac{1}{2}}$ are genuinely two-dimensional stencils which, in contrast to (1.11), allow us to incorporate information from both the normal and transverse directions,

$$
S_{i+\frac{1}{2}, j}:=\left\{\left(i^{\prime}, j^{\prime}\right)|| i^{\prime}-i-1 / 2|+| j^{\prime}-j \mid<q\right\}, \quad S_{i, j+\frac{1}{2}}:=\left\{\left(i^{\prime}, j^{\prime}\right)|| i^{\prime}-i|+| j^{\prime}-j-1 / 2 \mid<q\right\} .
$$

Thus, in contrast to the standard use of one-dimensional stencils in (1.11), we advocate here the use of twodimensional "clouds", as the basic stencil for conservative GMD schemes. We present such a family of GMD schemes in Section 2, based on the potential-based framework introduced in our recent papers [29,30].

\subsection{Divergence preserving schemes}

A major issue for the numerical approximation of multi-dimensional ideal MHD equations (1.1) is the divergence constraint (1.4), (1.6). Standard finite volume schemes may not preserve discrete versions of the constraint, leading to numerical instabilities $[17,44]$. Different approaches have been suggested to handle the divergence constraint in MHD codes and we mention three of the currently available approaches below.

(i) The projection method, $[6,8,10]$, is based on the Hodge decomposition of the solution $\mathbf{B}$ of (1.5): the corrected field $\mathbf{B}^{*}:=\mathbf{B}^{n}-\nabla \Delta^{-1} \operatorname{div}\left(\mathbf{B}^{n}\right)$ is divergence free. The method is rather expensive, however, as it requires a global elliptic solver together with a proper set of boundary conditions to be solved at every time step, e.g. [44].

(ii) The method of adding a source term, $[35,36]$, proportional to the divergence in (1.5) results in

$$
\mathbf{B}_{t}+\operatorname{curl}(\mathbf{B} \times \mathbf{u})=-\mathbf{u d i v}(\mathbf{B}) .
$$

The form (1.14) is symmetrizable [35]. A variant of this approach is found in the Generalized Lagrange multiplier method [13]. Applying the divergence to both sides, we obtain

$$
(\operatorname{div} \mathbf{B})_{t}+\operatorname{div}(\mathbf{u} \operatorname{div} \mathbf{B})=0 .
$$

Hence, any potential divergence errors are transported away from the computational domain by the flow. Recent papers $[17,19]$ have demonstrated that the added source term in (1.14) needs to be discretized in a very careful manner for numerical stability. Another difficulty with this approach lies in the non-conservative form of (1.14) which may result in wrong shock speeds [44].

(iii) The method of designing special divergence operators/staggering is a popular family of methods which consist of staggering the discretizations of the velocity and magnetic fields in (1.5). A wide variety of strategies for staggering the meshes has been proposed $[2-5,11,14,39,44]$ and references therein. The presence of different sets of meshes leads to problems when the staggered schemes are parallelized, boundary treatments, etc. Unstaggered variants of this approach have also been proposed in $[1,42,43]$.

The above discussion suggests that there is still a room for simple, computationally cheap finite volume schemes for the constrained equations that resolves GMD waves and preserve a discrete version of the associated constraint. In this paper, we present such a method with these desired properties for the divergence-free MHD equations. Our starting point are the GMD finite volume schemes proposed in [29,30]. These schemes modify standard finite volume fluxes by introducing vertex centered numerical potentials. The potentials incorporate explicit transverse information and lead to a stable and accurate resolution of genuinely multi-dimensional waves. A suitable choice of potentials results in GMD schemes that preserve constraints like divergence in the magnetic induction equation [29] or vorticity in the system of wave equations [30]. The schemes are very simple to implement and have low computational cost.

The extension of the potential-based GMD framework of [30] to the ideal MHD equations (1.1) is carried out in Section 2. A suitable choice of numerical potentials outlined in Section 3 is shown to yield schemes that preserves discrete divergence. Numerical experiments are reported in Section 4. 


\section{Genuinely multi-Dimensional (GMD) schemes}

Following the presentation of [30], we introduce the numerical potentials $\phi_{i+\frac{1}{2}, j+\frac{1}{2}}$ and $\psi_{i+\frac{1}{2}, j+\frac{1}{2}}$ at each vertex $\left(x_{i+\frac{1}{2}}, y_{j+\frac{1}{2}}\right)$, with the sole requirement that these potentials are consistent with the differential fluxes, i.e.,

$$
\phi_{i+\frac{1}{2}, j+\frac{1}{2}}(\mathbf{U}, \ldots, \mathbf{U})=\mathbf{f}(\mathbf{U}), \quad \psi_{i+\frac{1}{2}, j+\frac{1}{2}}(\mathbf{U}, \ldots, \mathbf{U})=\mathbf{g}(\mathbf{U})
$$

We need the following notation for standard averaging and (undivided) difference operators,

$$
\begin{aligned}
\mu_{x} a_{I, J}:=\frac{a_{I+\frac{1}{2}, J}+a_{I-\frac{1}{2}, J}}{2}, & \mu_{y} a_{I, J}:=\frac{a_{I, J+\frac{1}{2}}+a_{I, J-\frac{1}{2}}}{2}, \\
\delta_{x} a_{I, J}:=a_{I+\frac{1}{2}, J}-a_{I-\frac{1}{2}, J}, & \delta_{y} a_{I, J}:=a_{I, J+\frac{1}{2}}-a_{I, J-\frac{1}{2}} .
\end{aligned}
$$

A word about our notations: we note that the above discrete operators could be used with indexes $I, J$ which are placed at the center or at the edge of the computational cells, e.g., $I=i$ or $I=i+\frac{1}{2}$. In either case, we tag the resulting discrete operators according to the center of their stencil; thus, for example, $\mu_{x} w_{i+\frac{1}{2}}$ employs grid values placed on the integer-indexed edges, $w_{i}$ and $w_{i+1}$, whereas $\delta_{y} w_{j}$ employs the half-integer indexed centers, $w_{j \pm \frac{1}{2}}$.

We now set the numerical fluxes:

$$
\begin{aligned}
\mathbf{F}_{i+\frac{1}{2}, j} & =\mu_{y} \phi_{i+\frac{1}{2}, j}, \\
\mathbf{G}_{i, j+\frac{1}{2}} & =\mu_{x} \phi_{i, j+\frac{1}{2}} .
\end{aligned}
$$

The resulting finite volume scheme written in terms of the numerical potentials reads

$$
\begin{aligned}
\frac{\mathrm{d}}{\mathrm{d} t} \mathbf{U}_{i, j}= & -\frac{1}{\Delta x} \delta_{x} \mu_{y} \phi_{i, j}-\frac{1}{\Delta y} \delta_{y} \mu_{x} \psi_{i, j} \\
= & -\frac{1}{\Delta x}\left(\frac{1}{2}\left(\phi_{i+\frac{1}{2}, j+\frac{1}{2}}+\phi_{i+\frac{1}{2}, j-\frac{1}{2}}\right)-\frac{1}{2}\left(\phi_{i-\frac{1}{2}, j+\frac{1}{2}}+\phi_{i-\frac{1}{2}, j-\frac{1}{2}}\right)\right) \\
& -\frac{1}{\Delta y}\left(\frac{1}{2}\left(\psi_{i+\frac{1}{2}, j+\frac{1}{2}}+\psi_{i-\frac{1}{2}, j+\frac{1}{2}}\right)-\frac{1}{2}\left(\psi_{i+\frac{1}{2}, j-\frac{1}{2}}+\psi_{i-\frac{1}{2}, j-\frac{1}{2}}\right)\right) .
\end{aligned}
$$

The potential based scheme (2.3) is clearly conservative as well as consistent as the potentials $\phi, \psi$ are consistent. The genuinely multi-dimensional nature of the scheme is evident from (2.3): the potentials are differenced in the normal direction but averaged in the transverse direction. We claim that the family of potential-based schemes (2.3) is rich: any standard finite volume flux can be used as a building block for constructing the numerical potentials in (2.2), and the resulting potential-based scheme inherits the accuracy of the underlying numerical flux. There are several ways to pursue the construction of numerical potentials and we outline three of them below.

\subsection{Symmetric potentials}

In this approach, the potentials are defined by averaging the finite volume fluxes neighboring a vertex:

$$
\begin{aligned}
& \phi_{i+\frac{1}{2}, j+\frac{1}{2}}=\mu_{y} \mathbf{F}_{i+\frac{1}{2}, j+\frac{1}{2}}, \\
& \psi_{i+\frac{1}{2}, j+\frac{1}{2}}=\mu_{x} \mathbf{G}_{i+\frac{1}{2}, j+\frac{1}{2}},
\end{aligned}
$$

where $\mathbf{F}, \mathbf{G}$ are any numerical fluxes consistent with $\mathbf{f}$ and $\mathbf{g}$ respectively. An explicit computation of (2.3) with potentials (2.4) leads to the revealing form,

$$
\begin{aligned}
\frac{\mathrm{d}}{\mathrm{d} t} \mathbf{U}_{i, j}= & -\frac{1}{2 \Delta x}\left(\mu_{y} \mathbf{F}_{i+\frac{1}{2}, j+\frac{1}{2}}+\mu_{y} \mathbf{F}_{i+\frac{1}{2}, j-\frac{1}{2}}-\mu_{y} \mathbf{F}_{i-\frac{1}{2}, j+\frac{1}{2}}-\mu_{y} \mathbf{F}_{i-\frac{1}{2}, j-\frac{1}{2}}\right) \\
& -\frac{1}{2 \Delta y}\left(\mu_{x} \mathbf{G}_{i+\frac{1}{2}, j+\frac{1}{2}}+\mu_{x} \mathbf{G}_{i-\frac{1}{2}, j+\frac{1}{2}}-\mu_{x} \mathbf{G}_{i+\frac{1}{2}, j-\frac{1}{2}}-\mu_{x} \mathbf{G}_{i-\frac{1}{2}, j-\frac{1}{2}}\right) .
\end{aligned}
$$


Comparing the potential based scheme (2.5) with the standard finite volume scheme (1.7), we observe that the potential based scheme modifies (1.7) by averaging the fluxes in the transverse direction. Hence, it incorporates explicit transverse information in each direction. When employing two-point fluxes, the local stencil for the GMD scheme (2.5) consists of nine points instead of the standard five point stencil for the finite volume scheme (1.7). One can use wider stencils to achieve higher-order of accuracy; for example, the symmetric potential-based scheme based on second-order four-point MUSCL flux (1.12) yields a second-order GMD scheme based on a stencil of twenty-three points.

\subsection{Weighted symmetric potentials}

Weighted averages of the neighboring fluxes can be considered in place of the simple averaging used in (2.4). For prescribed $\theta_{i+\frac{1}{2}, j+\frac{1}{2}}, \kappa_{i+\frac{1}{2}, j+\frac{1}{2}} \in(0,1)$, the weighted potential is defined as

$$
\begin{aligned}
& \phi_{i+\frac{1}{2}, j+\frac{1}{2}}=\theta_{i+\frac{1}{2}, j+\frac{1}{2}} \mathbf{F}_{i+\frac{1}{2}, j+1}+\left(1-\theta_{i+\frac{1}{2}, j+\frac{1}{2}}\right) \mathbf{F}_{i+\frac{1}{2}, j}, \\
& \psi_{i+\frac{1}{2}, j+\frac{1}{2}}=\kappa_{i+\frac{1}{2}, j+\frac{1}{2}} \mathbf{G}_{i+1, j+\frac{1}{2}}+\left(1-\kappa_{i+\frac{1}{2}, j+\frac{1}{2}}\right) \mathbf{G}_{i, j+1 / 2} .
\end{aligned}
$$

The weights can be chosen based on the local characteristic speeds,

$$
\begin{aligned}
\theta_{i+\frac{1}{2}, j+\frac{1}{2}} & =\frac{\max \left\{-\left(\beta_{1}\right)_{i+\frac{1}{2}, j+\frac{1}{2}}, 0\right\}}{\max \left\{-\left(\beta_{1}\right)_{i+\frac{1}{2}, j+\frac{1}{2}}, 0\right\}+\max \left\{\left(\beta_{8}\right)_{i+\frac{1}{2}, j+\frac{1}{2}}, 0\right\}}, \\
\kappa_{i+\frac{1}{2}, j+\frac{1}{2}} & =\frac{\max \left\{-\left(\alpha_{1}\right)_{i+\frac{1}{2}, j+\frac{1}{2}}, 0\right\}}{\max \left\{-\left(\alpha_{1}\right)_{i+\frac{1}{2}, j+\frac{1}{2}}, 0\right\}+\max \left\{\left(\alpha_{8}\right)_{i+\frac{1}{2}, j+\frac{1}{2}}, 0\right\}} .
\end{aligned}
$$

Here, $\alpha_{l}$ and $, \beta_{l}, l=1,2, \ldots, N$ are the real eigenvalues of $A=\partial_{\mathbf{U}} \mathbf{f}\left(\mu_{y} \mu_{x} \mathbf{U}_{i+\frac{1}{2}, j+\frac{1}{2}}\right)$ and $B=$ $\partial_{\mathbf{U}} \mathbf{g}\left(\mu_{x} \mu_{y} \mathbf{U}_{i+\frac{1}{2}, j+\frac{1}{2}}\right)$, sorted in an increasing order. This choice of weights implies that the potential $(2.6)$ is "upwinded".

\subsection{Diagonal potentials}

We define the diagonal potentials [29],

$$
\begin{aligned}
& \phi_{i+\frac{1}{2}, j+\frac{1}{2}}=\frac{1}{2}\left(\mathbf{F}_{i+\frac{1}{2}, j+\frac{1}{2}}^{+}+\mathbf{F}_{i+\frac{1}{2}, j+\frac{1}{2}}^{-}\right), \\
& \psi_{i+\frac{1}{2}, j+\frac{1}{2}}=\frac{1}{2}\left(\mathbf{G}_{i+\frac{1}{2}, j+\frac{1}{2}}^{+}+\mathbf{G}_{i+\frac{1}{2}, j+\frac{1}{2}}^{-}\right) .
\end{aligned}
$$

Here, $\mathbf{F}^{ \pm}, \mathbf{G}^{ \pm}$are the diagonal fluxes

$$
\begin{array}{ll}
\mathbf{F}_{i+\frac{1}{2}, j+\frac{1}{2}}^{+}:=\mathbf{F}\left(\mathbf{U}_{i, j}, \mathbf{U}_{i+1, j+1}\right), & \mathbf{F}_{i+\frac{1}{2}, j-\frac{1}{2}}^{-}=\mathbf{F}\left(\mathbf{U}_{i, j}, \mathbf{U}_{i+1, j-1}\right) \\
\mathbf{G}_{i+\frac{1}{2}, j+\frac{1}{2}}^{+}:=\mathbf{G}\left(\mathbf{U}_{i, j}, \mathbf{U}_{i+1, j+1}\right), & \mathbf{G}_{i-\frac{1}{2}, j+\frac{1}{2}}^{-}:=\mathbf{G}\left(\mathbf{U}_{i, j}, \mathbf{U}_{i-1, j+1}\right) .
\end{array}
$$

which amount to rotating the $x$ - and $y$-axis by angles of $\frac{\pi}{4}$ and $-\frac{\pi}{4}$, where $\mathbf{F}(\cdot, \cdot)$ and $\mathbf{G}(\cdot, \cdot)$ are any two-point numerical fluxes consistent with $\mathbf{f}$ and $\mathbf{g}$.

\subsection{Isotropic GMD scheme}

We conclude our list for recipes of GMD schemes with an example which is not rendered by a numerical potential, but nevertheless, highlights the use of a GMD stencil. Let $\mathbf{F}(\cdot, \cdot)$ and $\mathbf{G}(\cdot, \cdot)$ are any two-point 
consistent numerical fluxes and let $\mathbf{F}^{ \pm}, \mathbf{G}^{ \pm}$be the corresponding diagonal numerical fluxes in (2.8b). We define the isotropic fluxes,

$$
\begin{aligned}
\mathcal{F}_{i+\frac{1}{2}, j} & :=\frac{1}{4}\left(\mathbf{F}_{i+\frac{1}{2}, j+\frac{1}{2}}^{+}+2 \mathbf{F}_{i+\frac{1}{2}, j}+\mathbf{F}_{i+\frac{1}{2}, j-\frac{1}{2}}^{-}\right), \\
\mathcal{G}_{i, j+\frac{1}{2}} & :=\frac{1}{4}\left(\mathbf{G}_{i+\frac{1}{2}, j+\frac{1}{2}}^{+}+2 \mathbf{G}_{i, j+\frac{1}{2}}+\mathbf{G}_{i-\frac{1}{2}, j+\frac{1}{2}}^{-}\right) .
\end{aligned}
$$

The resulting finite volume scheme reads as

$$
\begin{aligned}
\frac{\mathrm{d}}{\mathrm{d} t} \mathbf{U}_{i, j} & =-\frac{1}{\Delta x} \delta_{x} \mathcal{F}_{i, j}-\frac{1}{D y} \delta_{y} \mathcal{G}_{i, j}, \\
& =-\frac{1}{4 \Delta x}\left(\delta / \mathbf{F}_{i, j}^{+}+2 \delta_{x} \mathbf{F}_{i, j}+\delta \backslash \mathbf{F}_{i, j}^{-}\right)-\frac{1}{4 \Delta y}\left(\delta / \mathbf{G}_{i, j}^{+}+2 \delta_{y} \mathbf{G}_{i, j}-\delta \backslash \mathbf{G}_{i, j}^{-}\right) ;
\end{aligned}
$$

here, $\delta /$ and $\delta$ denote the diagonal difference operators,

$$
\delta / a_{I, J}:=a_{I+\frac{1}{2}, J+\frac{1}{2}}-a_{I-\frac{1}{2}, J-\frac{1}{2}}, \quad \delta \backslash a_{I, J}:=a_{I+\frac{1}{2}, J-\frac{1}{2}}-a_{I-\frac{1}{2}, J+\frac{1}{2}} .
$$

The GMD structure of the scheme is clear from (2.10): the scheme averages the fluxes along transverse directions. In contrast to the symmetric scheme (2.5), however, the explicit transverse information in (2.10) is obtained by "rotating" the fluxes. Since the scheme (2.10) is based on all nine grid points in a cell by involving all four directions, we term it as an isotropic GMD scheme. The isotropic GMD scheme (2.10) was shown to be entropy stable if the underlying two-point fluxes, $\mathbf{F}(\cdot, \cdot), \mathbf{G}(\cdot, \cdot)$ are, consult [30].

Second-order version of the isotropic schemes can be obtained by the piecewise bilinear reconstruction (1.12). Here, in addition to the point values reconstructed on (1.12e), we also need the corner point values,

$$
\begin{array}{ll}
\mathbf{U}_{i, j}^{N E}:=\mathbf{p}_{i, j}\left(x_{i+\frac{1}{2}}, y_{j+\frac{1}{2}}\right), & \mathbf{U}_{i, j}^{N W}:=\mathbf{p}_{i, j}\left(x_{i-\frac{1}{2}}, y_{j+\frac{1}{2}}\right), \\
\mathbf{U}_{i, j}^{S E}:=\mathbf{p}_{i, j}\left(x_{i+\frac{1}{2}}, y_{j-\frac{1}{2}}\right), & \mathbf{U}_{i, j}^{S W}:=\mathbf{p}_{i, j}\left(x_{i-\frac{1}{2}}, y_{j-\frac{1}{2}}\right),
\end{array}
$$

and the corresponding diagonal fluxes,

$$
\begin{aligned}
\mathbf{F}_{i+\frac{1}{2}, j+\frac{1}{2}}^{+}:=\mathbf{F}\left(\mathbf{U}_{i, j}^{N E}, \mathbf{U}_{i+1, j+1}^{S W}\right), & \mathbf{F}_{i+\frac{1}{2}, j-\frac{1}{2}}^{-}:=\mathbf{F}\left(\mathbf{U}_{i, j}^{S E}, \mathbf{U}_{i+1, j+1}^{N W}\right), \\
\mathbf{G}_{i+\frac{1}{2}, j+\frac{1}{2}}^{+}:=\mathbf{G}\left(\mathbf{U}_{i, j}^{N E}, \mathbf{U}_{i+1, j+1}^{S W}\right), & \mathbf{G}_{i-\frac{1}{2}, j+\frac{1}{2}}^{-}:=\mathbf{F}\left(\mathbf{U}_{i, j}^{N W}, \mathbf{U}_{i-1, j+1}^{S E}\right),
\end{aligned}
$$

to define the second order accurate version of the isotropic GMD scheme (2.10), (2.12).

\section{Divergence PRESERVING SCHEMES}

The divergence of the magnetic field in (1.1a) is preserved (1.4), due to the special structure of the MHD fluxes in (1.3). Accordingly, to ensure a divergence-free numerical solution we need to respect this special structure at the discrete level by choosing a suitable form of the potential. Let $\phi_{i+\frac{1}{2}, j+\frac{1}{2}}$ and $\psi_{i+\frac{1}{2}, j+\frac{1}{2}}$ be numerical potentials associated with the MHD fluxes $\mathbf{f}$ and $\mathbf{g}$; one may employ here any consistent potential such as the (weighted) symmetric, diagonal or isotropic potentials constructed in Section 2. Let $\eta$ and $\zeta$ be the reduced 6 -vector potentials consistent with the reduced fluxes $\widetilde{\mathbf{f}}$ and $\widetilde{\mathbf{g}}$ :

$$
\begin{aligned}
\eta_{i+\frac{1}{2}, j+\frac{1}{2}} & :=\left(\left(\phi_{1}\right)_{i+\frac{1}{2}, j+\frac{1}{2}}, \ldots,\left(\phi_{4}\right)_{i+\frac{1}{2}, j+\frac{1}{2}},\left(\phi_{7}\right)_{i+\frac{1}{2}, j+\frac{1}{2}},\left(\phi_{8}\right)_{i+\frac{1}{2}, j+\frac{1}{2}}\right)^{\top}, \\
\zeta_{i+\frac{1}{2}, j+\frac{1}{2}} & :=\left(\left(\psi_{1}\right)_{i+\frac{1}{2}, j+\frac{1}{2}}, \ldots,\left(\psi_{4}\right)_{i+\frac{1}{2}, j+\frac{1}{2}},\left(\psi_{7}\right)_{i+\frac{1}{2}, j+\frac{1}{2}},\left(\psi_{8}\right)_{i+\frac{1}{2}, j+\frac{1}{2}}\right)^{\top} .
\end{aligned}
$$


Our scheme will evolve the cell averages of the reduced vector $\widetilde{\mathbf{U}}:=\left(\rho, \rho u_{1}, \rho u_{2}, \rho u_{3}, B_{3}, E\right)^{\top}$ together with the $x$ - and $y$-components of the magnetic field, $B_{1}$ and $B_{2}$. The potential-based approximation of (1.3) reads

$$
\begin{aligned}
\frac{\mathrm{d}}{\mathrm{d} t} \widetilde{\mathbf{U}}_{i, j} & =-\frac{1}{\Delta x} \delta_{x} \mu_{y} \eta_{i, j}-\frac{1}{\Delta y} \delta_{y} \mu_{x} \zeta_{i, j}, \\
\frac{\mathrm{d}}{\mathrm{d} t}\left(B_{1}\right)_{i, j} & =-\frac{1}{\Delta y} \delta_{y} \mu_{x} \chi_{i, j}, \\
\frac{\mathrm{d}}{\mathrm{d} t}\left(B_{2}\right)_{i, j} & =\frac{1}{\Delta x} \delta_{x} \mu_{y} \chi_{i, j} .
\end{aligned}
$$

Here, $\chi_{i+\frac{1}{2}, j+\frac{1}{2}}$ is any numerical potential consistent with $h(\mathbf{U})=u_{1} B_{2}-u_{2} B_{1}$.

The divergence preserving property of (3.2) is summarized below.

Lemma 3.1. Let $\eta_{i+\frac{1}{2}, j+\frac{1}{2}}, \zeta_{i+\frac{1}{2}, j+\frac{1}{2}}$ and $\chi_{i+\frac{1}{2}, j+\frac{1}{2}}$ be arbitrary numerical potentials consistent with $\widetilde{\mathbf{f}}, \widetilde{\mathbf{g}}$ and, respectively, h. Let $\mathbf{B}_{i, j}$ be the approximate magnetic fields computed with the corresponding potential-based GMD scheme (3.2). Then, their discrete divergence div*,

$$
\operatorname{div}^{*}\left(\left(B_{1}, B_{2}\right)_{i, j}^{\top}\right):=\frac{1}{\Delta x} \mu_{y} \delta_{x}\left(B_{1}\right)_{i, j}+\frac{1}{\Delta y} \mu_{x} \delta_{y}\left(B_{2}\right)_{i, j}
$$

is preserved in time,

$$
\frac{\mathrm{d}}{\mathrm{d} t}\left(\operatorname{div}^{*}\left(\left(B_{1}, B_{2}\right)_{i, j}^{\top}\right)\right) \equiv 0, \quad \forall i, j
$$

Verification of $(3.3 \mathrm{~b})$ is straightforward: the difference operators $\delta_{x}, \delta_{y}$ and the averaging operators $\mu_{x}, \mu_{y}$ commute with each other. We apply the discrete divergence operator div* to the numerical scheme (3.2) to find

$$
\Delta x \Delta y \frac{\mathrm{d}}{\mathrm{d} t} \operatorname{div}^{*}\left(\left(B_{1}, B_{2}\right)_{i, j}^{\top}\right)=\left(\mu_{x} \delta_{y} \delta_{x} \mu_{y}-\mu_{y} \delta_{x} \delta_{x} \mu_{x}\right) \chi_{i, j} \equiv 0 .
$$

Remark 3.2. A similar treatment of divergence-preserving potential-based schemes for the 3D magnetic transport (1.5) was carried out in [29], Section 2.5. This could be served as the building block for divergence-preserving potential-based scheme for the 3D MHD equations along the lines of our treatment of 2D MHD equations in Lemma 3.1.

Remark 3.3. One approach in designing constraint preserving schemes is to satisfy that constraint approximately: for example, the discrete statement of the divergence constraint could be interpreted as a second-order approximation of the differential divergence,

$$
\operatorname{div}^{*}\left(\left(B_{1}, B_{2}\right)_{i, j}^{\top}\right)=\operatorname{div}\left(\left(B_{1}, B_{2}\right)^{\top}\right)\left(x_{i}, y_{j}\right)+\mathcal{O}\left(\Delta x^{2}+\Delta y^{2}\right) .
$$

This, however, requires the smoothness of the underlying solution. Instead, a key feature of constraint preserving schemes based on numerical potentials is that they satisfy exactly a discrete constraint, so that their numerical solution remains on a discrete sub-manifold, independent of the underlying smoothness. Similarly, a related potential-based GMD scheme which preserves a discrete vorticity was described in [30].

The scalar potential $\chi$ in (3.2) can be chosen in different ways. We mention two possible choices below.

\subsection{Divergence preserving symmetric GMD scheme}

The potentials $\eta, \zeta$ are defined as in (2.4). A natural choice [29] of the potential $\chi$ is the symmetric potential:

$$
\chi_{i+\frac{1}{2}, j+\frac{1}{2}}=\frac{1}{4}\left(\left(\mathbf{F}_{6}\right)_{i+\frac{1}{2}, j}+\left(\mathbf{F}_{6}\right)_{i+\frac{1}{2}, j+1}+\left(\mathbf{G}_{5}\right)_{i, j+\frac{1}{2}}+\left(\mathbf{G}_{5}\right)_{i+1, j+\frac{1}{2}}\right)
$$


with $\mathbf{F}_{5,6}, \mathbf{G}_{5,6}$ being components of any consistent numerical fluxes $\mathbf{F}, \mathbf{G}$. Let

$$
\widetilde{\mathbf{F}}=\left(\mathbf{F}_{1}, \ldots, \mathbf{F}_{4}, \mathbf{F}_{7}, \mathbf{F}_{8}\right)^{\top}, \quad \widetilde{\mathbf{G}}=\left(\mathbf{G}_{1}, \ldots, \mathbf{G}_{4}, \mathbf{G}_{7}, \mathbf{G}_{8}\right) \top
$$

be the corresponding reduced fluxes associated with any consistent F, G. The potential-based symmetric GMD scheme (3.2) takes the explicit form:

$$
\begin{aligned}
\frac{\mathrm{d}}{\mathrm{d} t} \widetilde{\mathbf{U}}_{i, j}= & -\frac{1}{2 \Delta x}\left(\mu_{y} \widetilde{\mathbf{F}}_{i+\frac{1}{2}, j+\frac{1}{2}}+\mu_{y} \widetilde{\mathbf{F}}_{i+\frac{1}{2}, j-\frac{1}{2}}-\mu_{y} \widetilde{\mathbf{F}}_{i-\frac{1}{2}, j+\frac{1}{2}}-\mu_{y} \widetilde{\mathbf{F}}_{i-\frac{1}{2}, j-\frac{1}{2}}\right) \\
& -\frac{1}{2 \Delta y}\left(\mu_{x} \widetilde{\mathbf{G}}_{i+\frac{1}{2}, j+\frac{1}{2}}+\mu_{x} \widetilde{\mathbf{G}}_{i-\frac{1}{2}, j+\frac{1}{2}}-\mu_{x} \widetilde{\mathbf{G}}_{i+\frac{1}{2}, j-\frac{1}{2}}-\mu_{x} \widetilde{\mathbf{G}}_{i-\frac{1}{2}, j-\frac{1}{2}}\right), \\
\frac{\mathrm{d}}{\mathrm{d} t}\left(B_{1}\right)_{i, j}= & -\frac{1}{4 \Delta y}\left(\mu_{x}\left(\mathbf{F}_{6}\right)_{i, j+1}-\mu_{x}\left(\mathbf{F}_{6}\right)_{i, j-1}\right)-\frac{1}{4 \Delta y}\left(\delta_{y}\left(\mu_{x}\left(\mathbf{G}_{5}\right)_{i+\frac{1}{2}, j+\frac{1}{2}}+\mu_{x}\left(\mathbf{G}_{5}\right)_{i-\frac{1}{2}, j+\frac{1}{2}}\right)\right), \\
\frac{\mathrm{d}}{\mathrm{d} t}\left(B_{2}\right)_{i, j}= & \frac{1}{4 \Delta x}\left(\mu_{y}\left(\mathbf{G}_{5}\right)_{i+1, j}-\mu_{y}\left(\mathbf{G}_{5}\right)_{i-1, j}\right)+\frac{1}{4 \Delta x}\left(\delta_{x}\left(\mu_{y}\left(\mathbf{F}_{6}\right)_{i+\frac{1}{2}, j+\frac{1}{2}}+\mu_{y}\left(\mathbf{F}_{6}\right)_{i+\frac{1}{2}, j-\frac{1}{2}}\right)\right) .
\end{aligned}
$$

\subsection{Divergence preserving isotropic GMD scheme}

We define a diagonal form of the potential $\chi$ :

$$
\chi_{i+\frac{1}{2}, j+\frac{1}{2}}=\frac{1}{4}\left(\left(\mathbf{F}_{6}^{+}\right)_{i+\frac{1}{2}, j+\frac{1}{2}}+\left(\mathbf{G}_{5}^{+}\right)_{i+\frac{1}{2}, j+\frac{1}{2}}+\left(\mathbf{F}_{6}^{-}\right)_{i+\frac{1}{2}, j+\frac{1}{2}}+\left(\mathbf{G}_{5}^{-}\right)_{i+\frac{1}{2}, j+\frac{1}{2}}\right)
$$

for diagonal fluxes $\mathbf{F}^{ \pm}, \mathbf{G}^{ \pm}$defined in (2.8b). Denote

$$
\widetilde{\mathbf{F}}^{ \pm}=\left(\mathbf{F}_{1}^{ \pm}, \ldots, \mathbf{F}_{4}^{ \pm}, \mathbf{F}_{7}^{ \pm}, \mathbf{F}_{8}^{ \pm}\right)^{\top}, \quad \widetilde{\mathbf{G}}^{ \pm}=\left(\mathbf{G}_{1}^{ \pm}, \ldots, \mathbf{G}_{4}^{ \pm}, \mathbf{G}_{7}^{ \pm}, \mathbf{G}_{8}^{ \pm}\right)^{\top} .
$$

The potential-based modification of the isotropic GMD scheme (2.10) based on the potential (3.6) reads

$$
\begin{aligned}
\frac{\mathrm{d}}{\mathrm{d} t} \widetilde{\mathbf{U}}_{i, j} & =-\frac{1}{4 \Delta x}\left(\delta / \widetilde{\mathbf{F}}_{i, j}^{+}+2 \delta_{x} \widetilde{\mathbf{F}}_{i, j}+\delta \widetilde{\mathbf{F}}_{i, j}^{-}\right)-\frac{1}{4 \Delta y}\left(\delta / \widetilde{\mathbf{G}}_{i, j}^{+}+2 \delta_{y} \widetilde{\mathbf{G}}_{i, j}-\delta \widetilde{\mathbf{G}}_{i, j}^{-}\right), \\
\frac{\mathrm{d}}{\mathrm{d} t}\left(B_{1}\right)_{i, j} & =-\frac{1}{4 \Delta y}\left(\mu_{x} \delta_{y}\left(\left(\mathbf{F}_{6}^{+}\right)_{i, j}+\left(\mathbf{F}_{6}^{-}\right)_{i, j}+\left(\mathbf{G}_{5}^{+}\right)_{i, j}+\left(\mathbf{G}_{5}^{-}\right)_{i, j}\right)\right), \\
\frac{\mathrm{d}}{\mathrm{d} t}\left(B_{2}\right)_{i, j} & =\frac{1}{4 \Delta x}\left(\mu_{y} \delta_{x}\left(\left(\mathbf{F}_{6}^{+}\right)_{i, j}+\left(\mathbf{F}_{6}^{-}\right)_{i, j}+\left(\mathbf{G}_{5}^{+}\right)_{i, j}+\left(\mathbf{G}_{5}^{-}\right)_{i, j}\right)\right) .
\end{aligned}
$$

Remark 3.4. Observe that the isotropic scheme (3.7) is not a potential-based scheme: the reduced vector $\widetilde{\mathbf{U}}_{i, j}$ is computed using the GMD isotropic fluxes. Instead, only the $x$ - and $y$-components of the magnetic field are evaluated using the diagonal-based potential $\chi_{i+\frac{1}{2}, j+\frac{1}{2}}$, consistent with $(1.3 \mathrm{~b}),(1.3 \mathrm{c})$ which in turn, imply the desired divergence-preserving property.

\section{NumericAl RESUlts}

All the potential based GMD schemes described in the previous section are semi-discrete. We define a fully discrete version of the first-order GMD schemes by using standard forward Euler time integration. Second-order strong stability preserving Runge-Kutta method [20] defines fully discrete versions of the second-order accurate GMD schemes. The time step is determined by a standard CFL condition. A CFL number of 0.45 is used in all the subsequent simulations. 


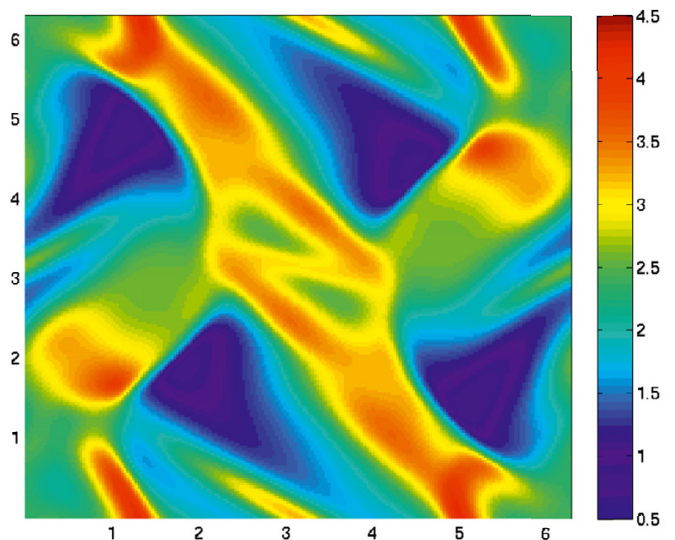

(a) SYM

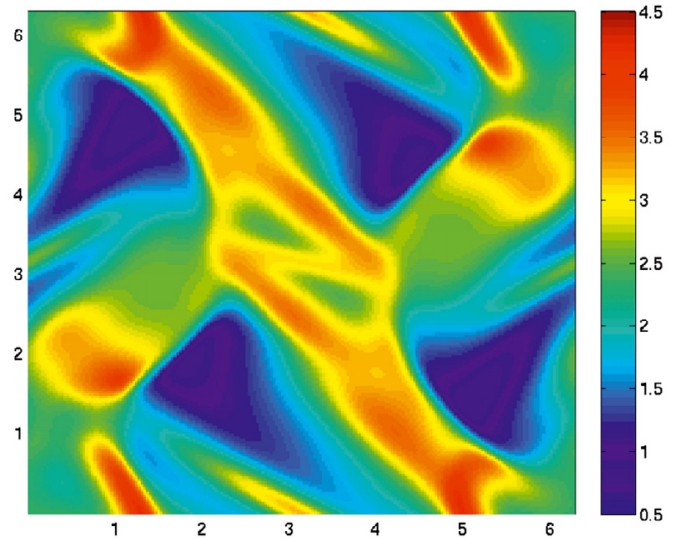

(c) ISO

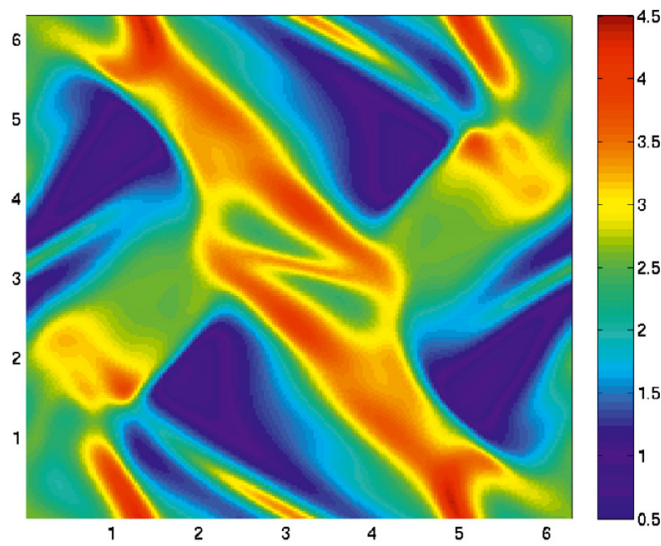

(b) SCP

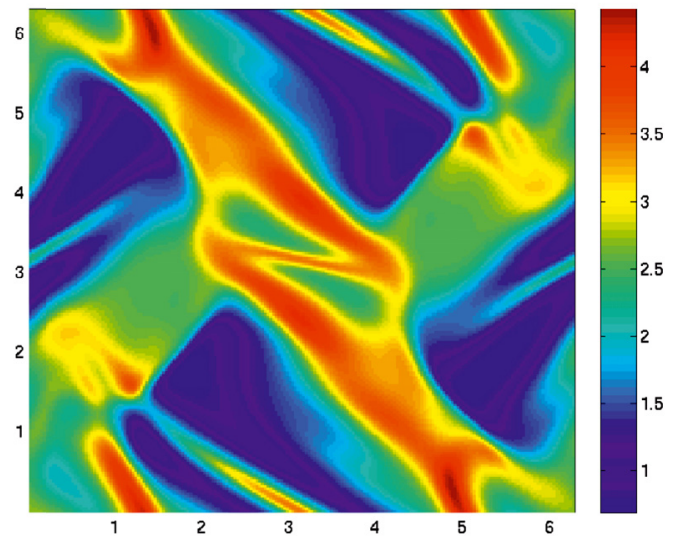

(d) ICP

Figure 1 . The pressure $p$ for the Orszag-Tang vortex computed at $t=\pi$ on a $200 \times 200$ mesh with first-order GMD schemes.

We test the following schemes:

SYM (SYM2) First (second)-order version of the symmetric GMD scheme (2.5).

ISO (ISO2) First (second)-order version of the isotropic GMD scheme (2.10).

SCP (SCP2) First (second)-order version of the divergence preserving symmetric GMD scheme (3.5).

ICP (ICP2) First (second)-order version of the divergence preserving isotropic GMD scheme (3.7).

\subsection{Orszag-Tang vortex}

The Orszag-Tang vortex is a widely reported benchmark for multi-dimensional MHD equations [44]. The initial data is

$$
\left(\rho, u_{1}, u_{2}, u_{3}, B_{1}, B_{2}, B_{3}, p\right)=\left(\gamma^{2},-\sin (y), \sin (x), 0,-\sin (y), \sin (2 x), 0, \gamma\right),
$$

in the computational domain: $(x, y, t) \in[0,2 \pi]^{2} \times[0, \pi]$ with periodic boundary conditions.

Although the exact solution is not known, qualitative features have been reported [44]. The solution consists of shocks along the diagonals and interesting smooth features including a vortex near the center of the domain. The approximate pressures, computed on a $200 \times 200$ mesh, are shown in Figures 1 and 2 . 


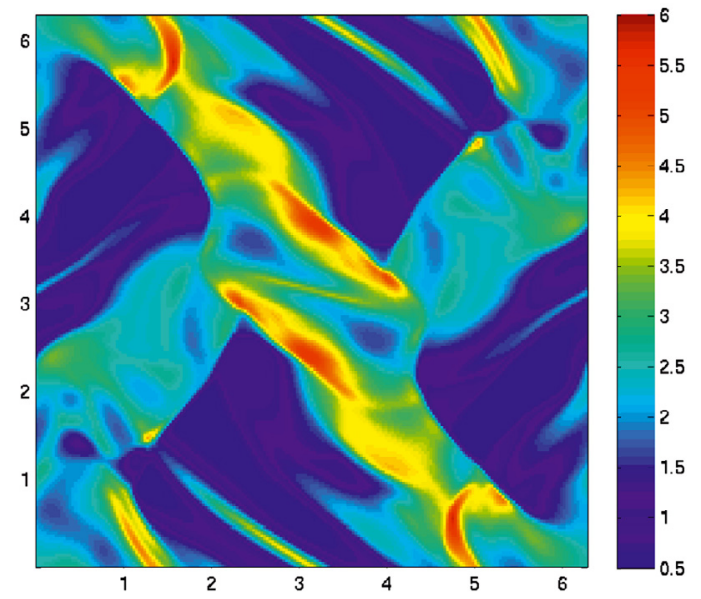

(a) SYM2

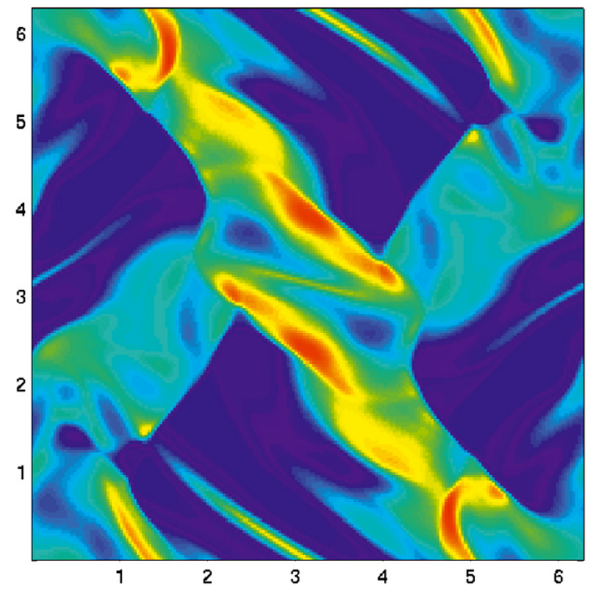

(c) $\mathrm{ISO} 2$

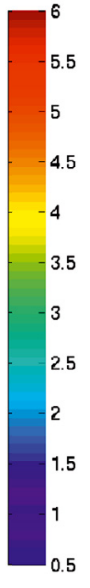

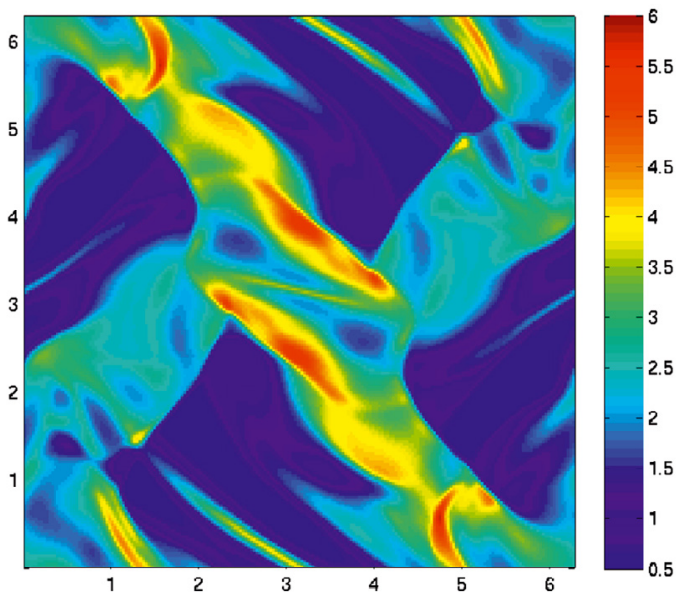

(b) SCP2

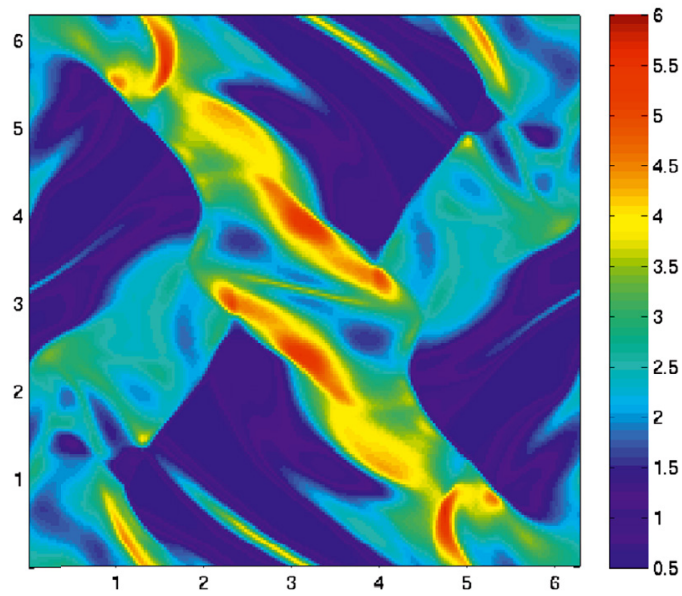

(d) ICP2

Figure 2. The pressure $p$ for the Orszag-Tang vortex computed at $t=\pi$ on a $200 \times 200$ mesh with second-order GMD schemes.

Figure 1 shows the approximate pressure computed with the first-order GMD schemes. The solution is smeared at this resolution, but the qualitative features are captured without any spurious oscillations and other numerical artifacts. The divergence preserving SCP and ICP schemes are clearly more accurate than the SYM and ISO schemes, indicating that preserving a discrete version of the constraint leads to a gain in accuracy. The results for the second-order schemes are plotted in Figure 2 and show a considerable improvement in the resolution. The gain in accuracy is pronounced, both at the shocks and at the central vortex. The divergence preserving SCP2 and ICP2 are slightly more accurate than the SYM2 and ISO2 schemes.

In the absence of an exact formula for the solution, the maximum pressure $[17,44]$ has been suggested as a measure of accuracy. The maximum pressure at time $t=\pi$, computed on a sequence of meshes, is presented in Table 1. The table provides a quantitative comparison between the schemes and vindicates the conclusions from the plots. The gain in resolution with the second-order schemes is considerable.

As the initial data is divergence free, the divergence constraint (1.6) implies that the divergence should remain zero during the evolution. We show the errors in the discrete divergence operator $\operatorname{div}^{*}(3.3 \mathrm{a})$, measured in the 
TABLE 1. Maximum pressure for the Orszag-Tang vortex with all the GMD schemes on a $M \times M$ mesh at time $t=\pi$.

\begin{tabular}{c|cccccccc}
\hline$M$ & SYM & ISO & SCP & ICP & SYM2 & ISO2 & SCP2 & ICP2 \\
\hline 50 & 3.17 & 3.19 & 3.27 & 3.32 & 4.85 & 4.34 & 4.89 & 4.37 \\
100 & 3.54 & 3.57 & 3.65 & 3.71 & 4.91 & 5.00 & 5.1 & 5.14 \\
200 & 4.24 & 4.22 & 4.37 & 4.42 & 5.75 & 5.64 & 5.76 & 5.71 \\
400 & 4.78 & 4.79 & 4.94 & 5.00 & 6.03 & 6.1 & 6.08 & 6.15 \\
\hline
\end{tabular}

TABle 2. Discrete divergence $\operatorname{div}^{*}(3.3 \mathrm{a})$ in $L^{1}$ for the Orszag-Tang vortex with all the GMD schemes on a $M \times M$ mesh at time $t=\pi$.

\begin{tabular}{c|cccccccc}
\hline$M$ & SYM & ISO & SCP & ICP & SYM2 & ISO2 & SCP2 & ICP2 \\
\hline 50 & 0.60 & 0.49 & $5.58 \mathrm{e}-14$ & $4.43 \mathrm{e}-14$ & 1.78 & 1.66 & $8.55 \mathrm{e}-14$ & $5.73 \mathrm{e}-14$ \\
100 & 0.89 & 0.71 & $2.23 \mathrm{e}-13$ & $1.78 \mathrm{e}-13$ & 3.26 & 2.99 & $3.16 \mathrm{e}-13$ & $1.99 \mathrm{e}-13$ \\
200 & 0.90 & 0.75 & $7.97 \mathrm{e}-13$ & $5.85 \mathrm{e}-13$ & 3.87 & 3.58 & $2.88 \mathrm{e}-13$ & $6.49 \mathrm{e}-13$ \\
400 & 0.81 & 0.70 & $2.51 \mathrm{e}-12$ & $1.82 \mathrm{e}-12$ & 3.88 & 3.32 & $3.27 \mathrm{e}-12$ & $1.93 \mathrm{e}-12$ \\
\hline
\end{tabular}

TABle 3. Discrete divergence $\operatorname{div}^{c}(4.1)$ in $L^{1}$ for the Orszag-Tang vortex with all the GMD schemes on a $M \times M$ mesh at time $t=\pi$.

\begin{tabular}{c|cccccccc}
\hline$M$ & SYM & ISO & SCP & ICP & SYM2 & ISO2 & SCP2 & ICP2 \\
\hline 50 & 0.62 & 0.49 & 0.18 & 0.27 & 2.43 & 2.09 & 1.20 & 1.55 \\
100 & 0.89 & 0.71 & 0.17 & 0.26 & 4.09 & 3.44 & 1.38 & 1.69 \\
200 & 0.90 & 0.75 & 0.14 & 0.20 & 4.59 & 4.02 & 1.23 & 1.57 \\
400 & 0.82 & 0.70 & 0.10 & 0.13 & 4.60 & 3.63 & 1.10 & 1.48 \\
\hline
\end{tabular}

$L^{1}$ norm, in Table 2. The table shows that the standard GMD schemes lead to $\mathcal{O}(1)$ divergence errors. The divergence error is larger for the second-order SYM2 and ISO2 schemes than the first-order schemes. This is to be expected as the second-order schemes resolve the shocks sharply. On the other hand the SCP, SCP2, ICP and ICP2 schemes preserve this discrete divergence to machine precision.

It is natural to question whether controlling one discrete version of divergence, div*, will imply control of a different discrete version of the divergence operator. The standard central discrete divergence operator is defined by,

$$
\operatorname{div}^{c}\left(\left(B_{1}, B_{2}\right)_{i, j}^{\top}\right):=\frac{1}{\Delta x} \delta_{x}\left(B_{1}\right)_{i, j}+\frac{1}{\Delta y} \delta_{y}\left(B_{2}\right)_{i, j}
$$

Note that the central discrete divergence operator $\operatorname{div}^{c}$ differs from the discrete divergence operator div* by $\mathcal{O}\left(\Delta x^{2}+\Delta y^{2}\right)$ provided that the underlying magnetic field is smooth. However, since the magnetic field in this example has shocks and other discontinuities, it is not clear that preserving div* will lead to some control in $\operatorname{div}^{c}$. We explore this question by presenting errors in $\operatorname{div}^{c}$ at time $t=\pi$ on a sequence of meshes in Table 3.

A closer investigation of Table 3 and comparison between Tables 2 and 3 reveal an interesting picture. As with div ${ }^{*}$ errors, the errors in the discrete divergence $\operatorname{div}^{c}$ increase when the second-order schemes are used. Furthermore, the errors for both discrete divergence operators with the first- and second-order SYM and ISO schemes are of comparable magnitude. On the other hand, controlling div* errors to machine precision does not imply that the errors in $\operatorname{div}^{c}$ with the SCP and ICP schemes are very low. In fact, the errors are $\mathcal{O}(1)$ due to the formation of discontinuities in this problem.

However, the errors in $\operatorname{div}^{c}$ with the SCP and ICP schemes are consistently lower than the corresponding errors with the SYM and ISO schemes. The difference ranges from a factor of about four for the second-order schemes to almost an order of magnitude for the first-order schemes. Furthermore, the errors with the SCP and 


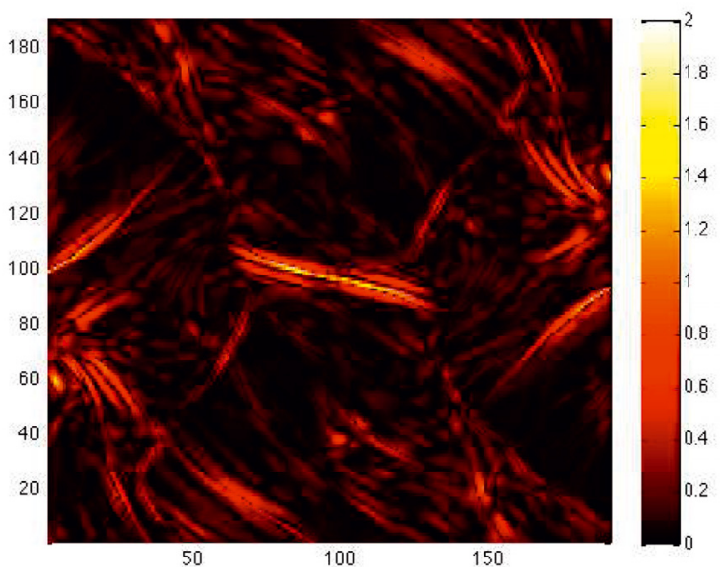

(a) SYM2

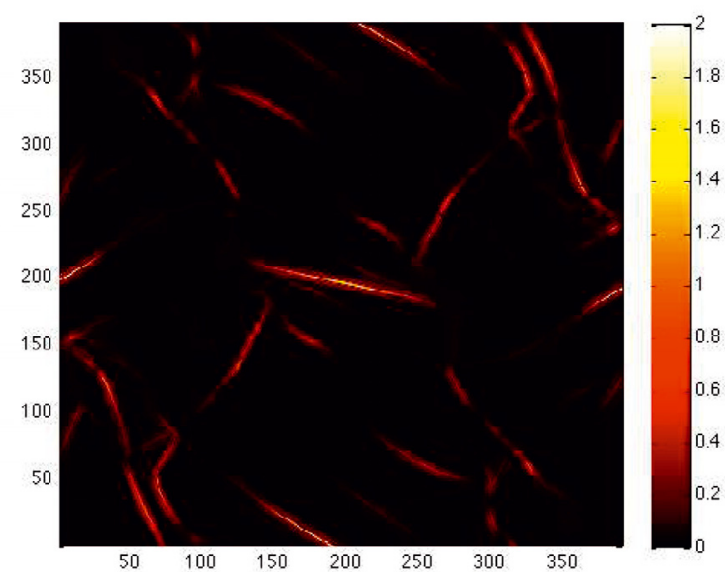

(b) $\mathrm{SCP} 2$

Figure 3. The discrete divergence $\operatorname{div}^{c}$ (4.1) for the Orszag-Tang vortex computed at $t=\pi$ on a $400 \times 400$ mesh with second-order SYM2 and SCP2 schemes.

ICP schemes are reduced by decreasing the mesh size indicating some convergence (but at a very slow rate) as the mesh is refined.

In-order to explain the above observations, we plot the discrete divergence $\operatorname{div}^{c}$ for the SYM2 and SCP2 schemes on a $400 \times 400$ mesh in Figure 3. The figure clearly shows that errors in the discrete divergence operator $\operatorname{div}^{c}$ with the SCP2 scheme are created at shocks and sharp gradients in the solution, see Figure 2 for a comparison. However, the errors in the SYM2 scheme are not localized. The largest magnitude of error is still at the discontinuities but there are large errors away from them. Figure 3 and Tables 2 and 3 suggest that preserving one version of discrete divergence implies leads to some control on another version of discrete divergence in the smooth parts of the solution and localization of divergence errors at shocks. Therefore, the overall divergence error with the SCP and ICP schemes is lower than that of the SYM and ISO schemes.

\subsection{Rotor problem}

Another benchmark test for the MHD equations is the rotor problem [44]. The computational domain is $(x, y, t) \in[0,1]^{2} \times[0,0.295]$ with artificial Neumann type boundary conditions. The initial density is

$$
\rho= \begin{cases}10.0 & \text { if } r<0.1 \\ 1+9 f(r) & \text { if } 0.1 \leq r<0.115 \\ 1.0 & \text { otherwise }\end{cases}
$$

with $r(x, y)=|(x, y)-(0.5,0.5)|$ and

$$
f(r)=\frac{23-200 r}{3}
$$

The other initial variables are,

$$
\begin{aligned}
\left(\rho u_{1}, \rho u_{2}\right) & = \begin{cases}((10 y-5) \rho,-(10 x-5) \rho) & \text { if } r<0.1, \\
((10 y-5) f(r) \rho,-(10 x-5) f(r) \rho) & \text { if } 0.1 \leq r<0.115, \\
(0.0,0.0) & \text { otherwise }\end{cases} \\
\left(\rho u_{3}, B_{1}, B_{2}, B_{3}, p\right) & =(0.0,2.5 / \sqrt{\pi}, 0.0,0.0,0.5) .
\end{aligned}
$$




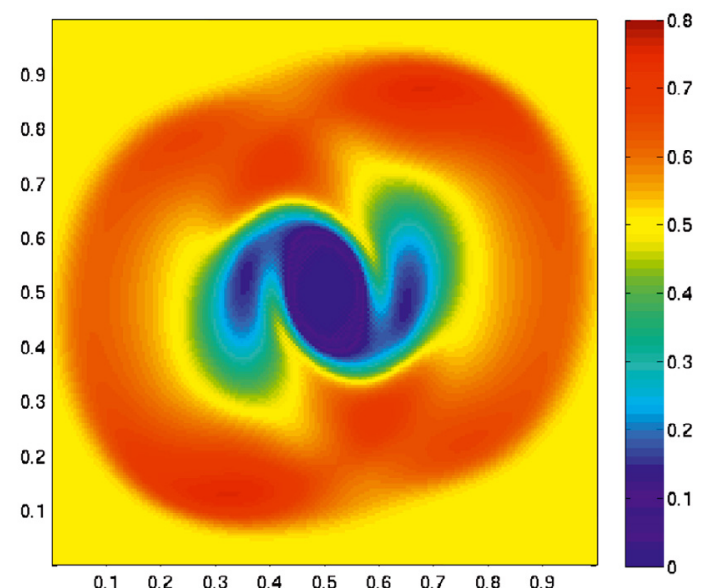

(a) SYM

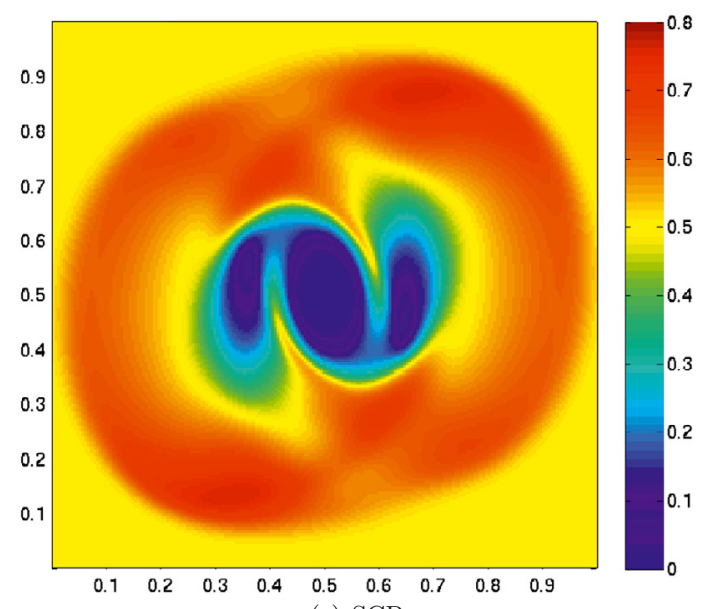

(c) SCP

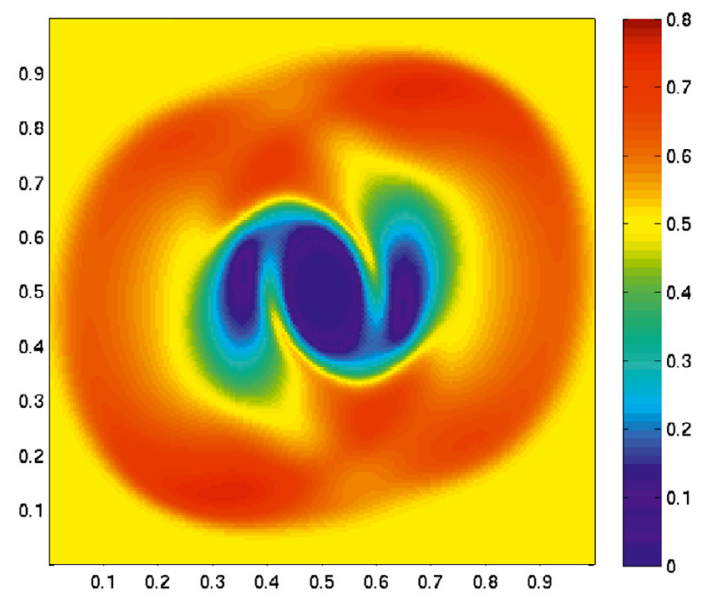

(b) ISO

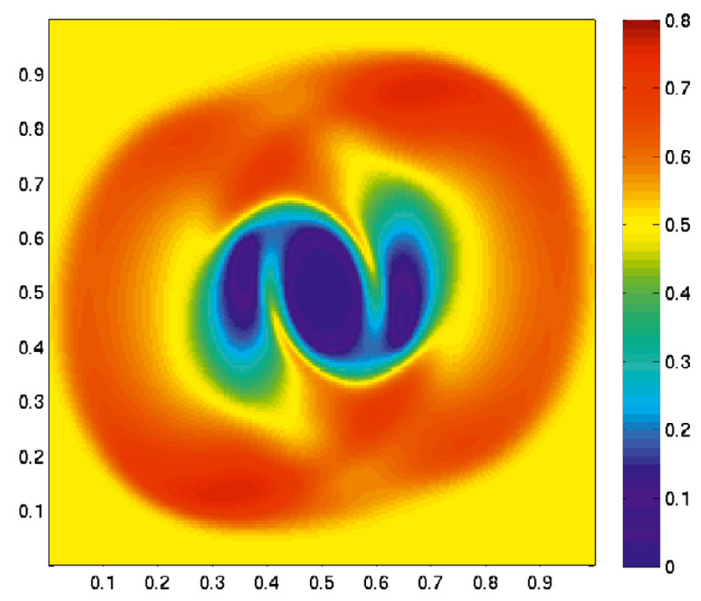

(d) ICP

Figure 4 . The pressure $p$ for the rotor problem computed at $t=0.295$ on a $200 \times 200$ mesh with first-order schemes.

The initial velocity and magnetic fields are such that the variables are rotated in the domain. The pressure drops to very low values in the center, and this test case is set up in order to determine how a scheme handles low pressures. The approximate pressure computed with the first-order GMD schemes, on a $200 \times 200$ mesh, is shown in Figure 4. The figure shows that all the schemes are stable at this resolution and the low pressure at the center is resolved. The first-order schemes are diffusive. The divergence preserving SCP and ICP schemes are more accurate in this case. The results for the second-order schemes are plotted in Figure 5. They reveal a significant gain in resolution with the second-order schemes, particularly at shocks. The errors in the discrete divergence div $^{*}$ are displayed in Table 4 . The divergence errors generated by the SYM and ISO schemes and their second-order versions are again $\mathcal{O}(1)$. These errors increase with increasing resolution, i.e., either by reducing mesh size or by increasing the order of accuracy of the scheme, indicating that the bulk of the divergence errors are generated near the shocks. The SCP, ICP, SCP2 and ICP2 schemes preserve discrete divergence to machine precision. 
TABle 4. Discrete divergence $\operatorname{div}^{*}(3.3 \mathrm{a})$ in $L^{1}$ for the rotor problem with all the eight schemes on a $M \times M$ mesh at time $t=0.295$.

\begin{tabular}{c|cccccccc}
\hline$M$ & SYM & ISO & SCP & ICP & SYM2 & ISO2 & SCP2 & ICP2 \\
\hline 50 & 0.70 & 0.57 & $6.0 \mathrm{e}-12$ & $4.49 \mathrm{e}-12$ & 1.03 & 1.03 & $6.3 \mathrm{e}-13$ & $4.7 \mathrm{e}-13$ \\
100 & 1.25 & 0.93 & $2.3 \mathrm{e}-12$ & $1.73 \mathrm{e}-12$ & 1.92 & 1.9 & $2.4 \mathrm{e}-13$ & $1.8 \mathrm{e}-13$ \\
200 & 1.7 & 1.23 & $8.8 \mathrm{e}-13$ & $6.35 \mathrm{e}-13$ & 3.41 & 3.27 & $4.1 \mathrm{e}-13$ & $1.6 \mathrm{e}-13$ \\
400 & 2.09 & 1.56 & $3.3 \mathrm{e}-13$ & $2.49 \mathrm{e}-13$ & 6.0 & 5.7 & $4.2 \mathrm{e}-13$ & $1.1 \mathrm{e}-13$ \\
\hline
\end{tabular}

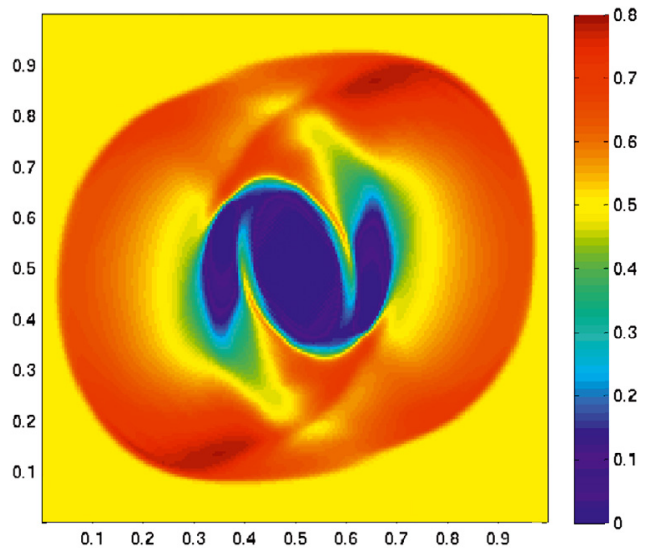

(a) SYM2

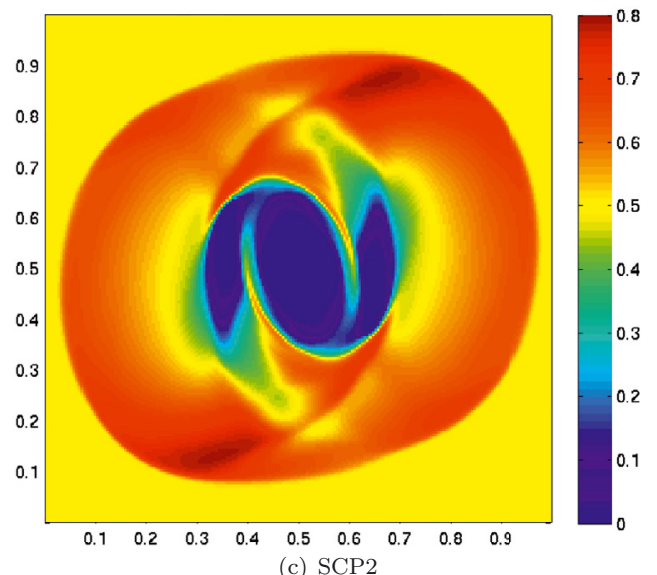

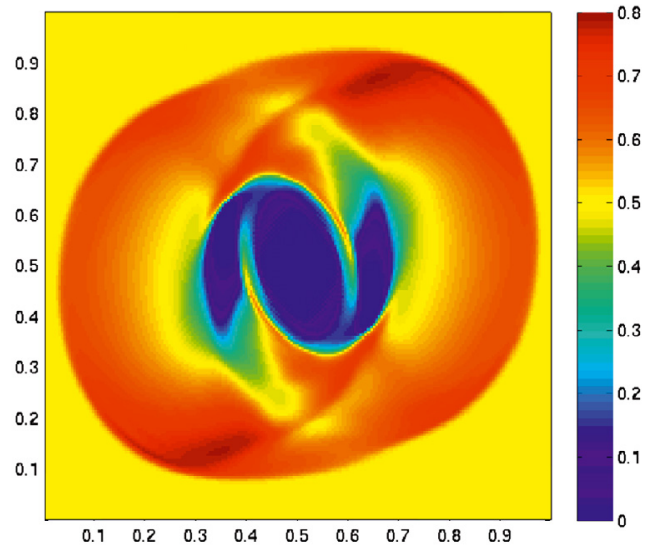

(b) ISO2

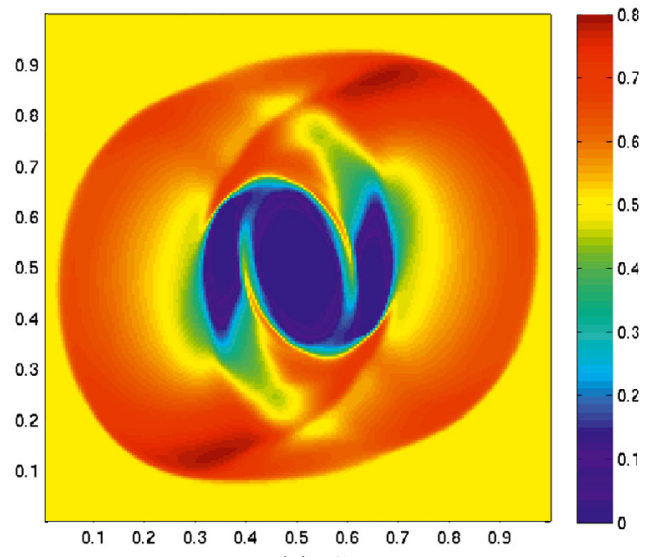

(d) ICP2

FiguRE 5. The pressure $p$ for the rotor problem computed at $t=0.295$ on a $200 \times 200$ mesh with second-order schemes.

\subsection{Cloud-Shock interaction}

The next benchmark test case for the MHD equations involves the interaction of a high density cloud with a shock. The initial data for this cloud-shock interaction problem [38] consists of a shock located at $x=0.05$ with

$$
\left(\rho, u_{1}, u_{2}, u_{3}, B_{1}, B_{2}, B_{3}, p\right)= \begin{cases}(3.86859,11.2536,0,0,0,2.1826182,-2.1826182,167.345), & \text { if } x<0.05 \\ (1.0,0,0,0,0,0.56418958,0.56418958,1.0), & \text { if } x<0.05\end{cases}
$$




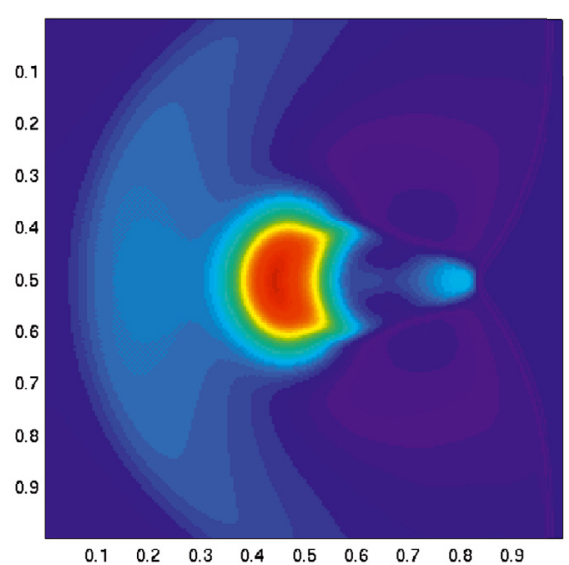

(a) SYM

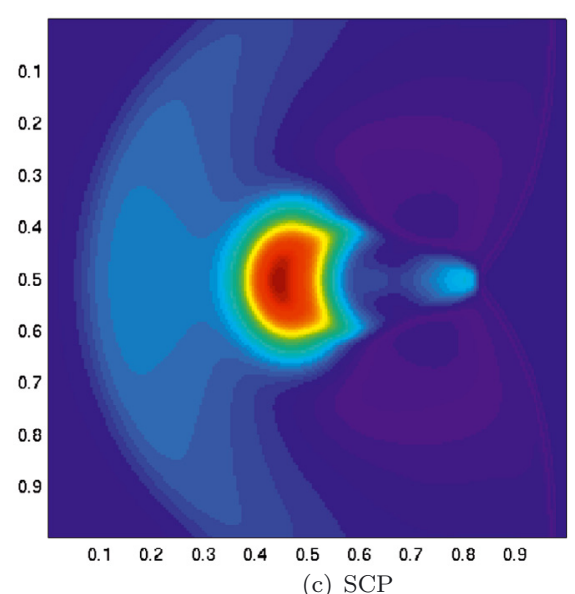

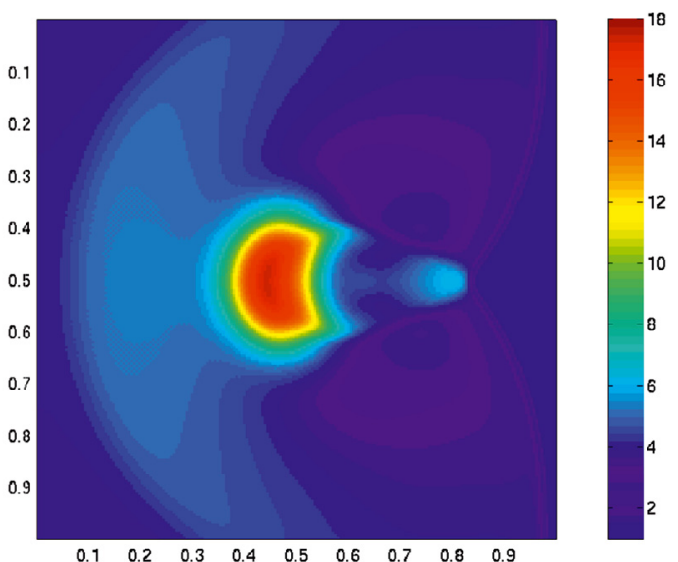

(b) ISO

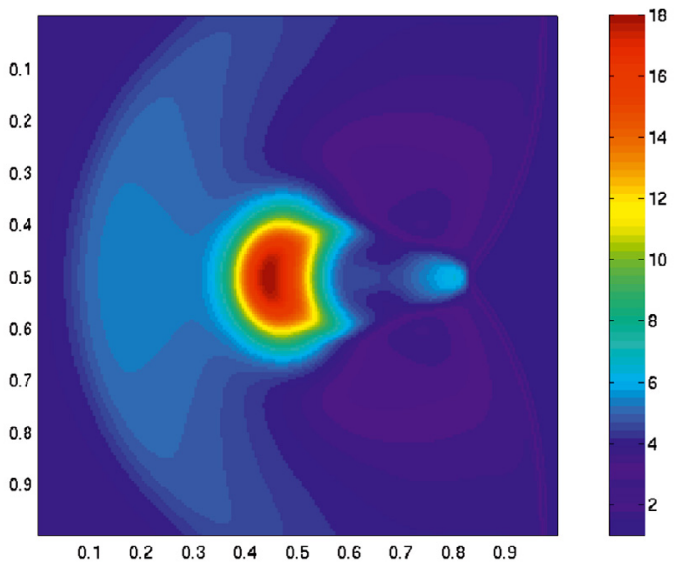

(d) ICP

Figure 6. The density $\rho$ for the cloud-shock interaction computed at $t=0.06$ on a $200 \times 200$ mesh with first-order schemes.

and a circular cloud of density $\rho=10$ with radius 0.15 , centered at $(x, y)=(0.25,0.5)$. The computational domain is $[0,1] \times[0,1]$. The test is configured in such a way that a right moving shock violently interacts with a high density cloud. The solution has a extremely complex structure, consisting of bow shock at the left, trailing shocks at the right and a complicated smooth region with turbulent features in the center.

We plot the approximate density, on a $200 \times 200$ mesh, at time $t=0.06$ in Figures 6 and 7 .

The first-order results in Figure 6 show that the first-order GMD schemes are stable but quite diffusive. The divergence preserving SCP and ICP schemes are again more accurate than the SYM and ISO schemes. The second-order results are plotted in Figure 7 and show a dramatic increase in resolution. Both the bow shock and the trailing shock are captured accurately. The smooth region with turbulent like features is also resolved quite well. The divergence errors for discrete divergence div* are shown in Table 5. The table shows large divergence errors for the SYM (SYM2) and ISO (ISO2) schemes. On the other hand, the constraint preserving SCP (SCP2) and ICP (ICP2) schemes preserve discrete divergence to machine precision.

Remark 4.1. The first-order GMD schemes were quite diffusive. A possible reason is the use of the Rusanov flux (1.9). This flux is known to produce excessive smearing at the shocks. However, we advocate the use of the 
TABle 5. Discrete divergence $\operatorname{div}^{*}(3.3 \mathrm{a})$ in $L^{1}$ for cloud shock interaction with all the eight schemes on a $M \times M$ mesh at time $t=0.06$.

\begin{tabular}{c|cccccccc}
\hline$M$ & SYM & ISO & SCP & ICP & SYM2 & ISO2 & SCP2 & ICP2 \\
\hline 50 & 4.56 & 2.59 & $2.8 \mathrm{e}-12$ & $2.1 \mathrm{e}-12$ & 5.79 & 5.38 & $3.4 \mathrm{e}-13$ & $2.27 \mathrm{e}-13$ \\
100 & 4.47 & 3.3 & $1.2 \mathrm{e}-12$ & $8.7 \mathrm{e}-13$ & 12.58 & 11.75 & $2.1 \mathrm{e}-13$ & $1.14 \mathrm{e}-13$ \\
200 & 5.19 & 4.05 & $5.0 \mathrm{e}-13$ & $3.7 \mathrm{e}-13$ & 27.1 & 26.48 & $1.4 \mathrm{e}-13$ & $1.34 \mathrm{e}-13$ \\
400 & 7.5 & 6.4 & $2.3 \mathrm{e}-13$ & $1.5 \mathrm{e}-13$ & 38.0 & 41.3 & $1.8 \mathrm{e}-13$ & $2.2 \mathrm{e}-13$ \\
\hline
\end{tabular}

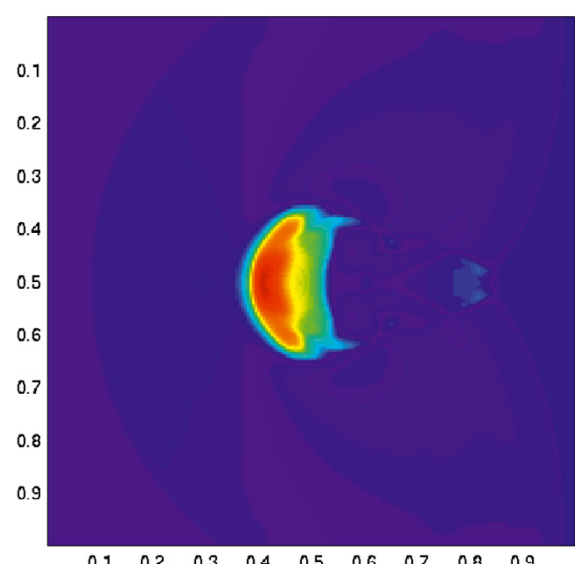

(a) SYM2

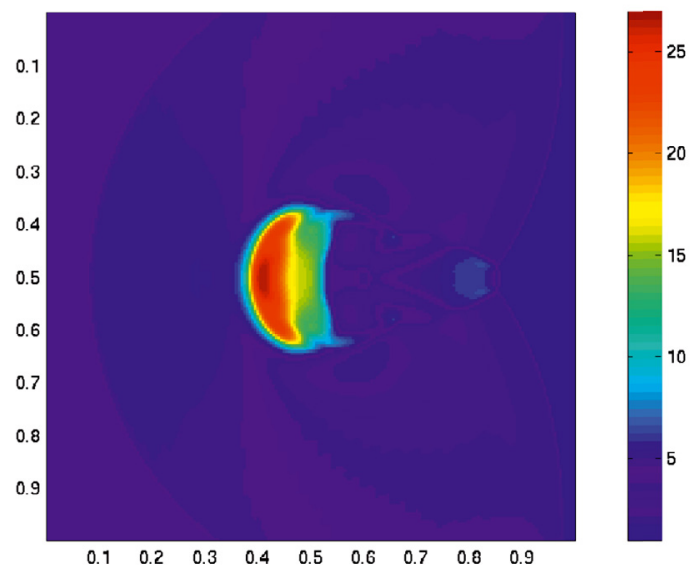

(c) $\mathrm{ISO} 2$

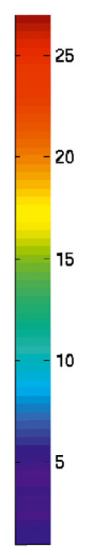

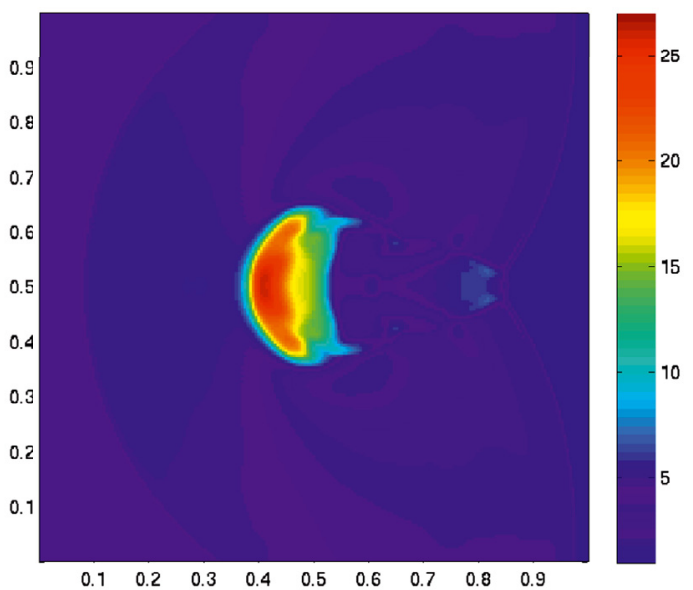

(b) $\mathrm{SCP} 2$

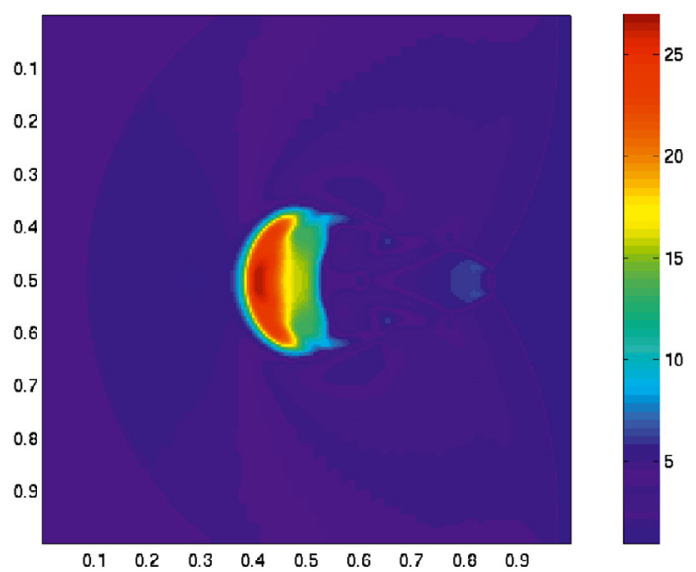

(d) ICP2

Figure 7. The density $\rho$ for the cloud-shock interaction computed at $t=0.06$ on a $200 \times 200$ mesh with second-order schemes.

Rusanov flux as the accuracy is recovered at second-order. The Rusanov flux is very easy to implement, uses minimal characteristic information and has a low computational cost. It fits into the black box framework of our GMD schemes.

The three numerical experiments show that the GMD schemes are quite robust. There does not appear to be a strong connection between the divergence errors and stability of a GMD scheme. The GMD structure of the 
schemes incorporates stability. However, there is a gain in accuracy (at least at first-order) when the divergence preserving versions of the scheme are used.

Physicists are generally reluctant to use numerical schemes that produce divergence errors. Hence, we advocate the use of the divergence preserving GMD schemes. Furthermore, the computational cost of a divergence preserving GMD scheme is virtually identical to the cost of other GMD schemes.

\section{Conclusion}

The ideal MHD equations (1.1a) are considered. The equations are non-strictly hyperbolic and posses a complex shock structure. Design of stable and accurate numerical methods for the MHD equations in multidimensions is complicated on account of its genuinely multi-dimensional structure and the divergence constraint.

We extend the potential based GMD framework of recent papers $[29,30]$ to the MHD equations. The finite volume schemes are formulated in terms of vertex centered numerical potentials. Symmetric (2.5) and isotropic (2.10) versions of the potential based GMD schemes are described. The GMD schemes are modified with a suitable choice of potentials to yield divergence preserving GMD schemes. Second-order versions are obtained by employing non-oscillatory piecewise bilinear reconstructions. The schemes are constraint preserving GMD extensions of the central schemes of Kurganov and Tadmor [23].

Benchmark numerical experiments for the MHD equations are presented. They show that the first-order GMD schemes resolve the waves with some diffusion. There is a gain in accuracy when the divergence preserving versions are used. The gain in resolution with the second-order schemes is considerable. The multi-dimensional shocks are vortices are captured, with good accuracy.

The non divergence preserving versions of the GMD schemes can generate large divergence errors, particularly at shocks. These errors do not seem to affect the stability of the schemes, at least in our tests. But large divergence errors might create instabilities at finer resolutions. Hence, we advocate using the divergence preserving versions of the GMD schemes. The GMD approach is simple to implement, robust and has a very low computational cost. It will be extended to higher than second-order of accuracy and to unstructured meshes in future papers. Other future projects include using the divergence preserving GMD schemes to compute realistic flows in solar physics and astrophysics.

\section{REFERENCES}

[1] R. Artebrant and M. Torrilhon, Increasing the accuracy of local divergence preserving schemes for MHD. J. Comput. Phys. 227 (2008) 3405-3427.

[2] J. Bálbas and E. Tadmor, Non-oscillatory central schemes for one and two-dimensional magnetohydrodynamics II: High-order semi-discrete schemes. SIAM. J. Sci. Comput. 28 (2006) 533-560.

[3] J. Bálbas, E. Tadmor and C.C. Wu, Non-oscillatory central schemes for one and two-dimensional magnetohydrodynamics I. J. Comput. Phys. 201 (2004) 261-285.

[4] D.S. Balsara, Divergence free adaptive mesh refinement for magnetohydrodynamics. J. Comput. Phys. 174 (2001) 614-648.

[5] D.S. Balsara and D. Spicer, A staggered mesh algorithm using high order Godunov fluxes to ensure solenoidal magnetic fields in magnetohydrodynamic simulations. J. Comput. Phys. 149 (1999) 270-292.

[6] J.B. Bell, P. Colella and H.M. Glaz, A second-order projection method for the incompressible Navier-Stokes equations. J. Comput. Phys. 85 (1989) 257-283.

[7] F. Bouchut, C. Klingenberg and K. Waagan, A multi-wave HLL approximate Riemann solver for ideal MHD based on relaxation I- theoretical framework. Numer. Math. 108 (2007) 7-42.

[8] J.U. Brackbill and D.C. Barnes, The effect of nonzero Div $B$ on the numerical solution of the magnetohydrodynamic equations. J. Comput. Phys. 35 (1980) 426-430.

[9] M. Brio and C.C. Wu, An upwind differencing scheme for the equations of ideal MHD. J. Comput. Phys. 75 (1988) 400-422.

[10] A.J. Chorin, Numerical solutions of the Navier-Stokes equations. Math. Comput. 22 (1968) 745-762.

[11] W. Dai and P.R. Woodward, A simple finite difference scheme for multi-dimensional magnetohydrodynamic equations. J. Comput. Phys. 142 (1998) 331-369.

[12] H. Deconnik, P.L. Roe and R. Struijs, A multi-dimensional generalization of Roe's flux difference splitter for Euler equations. Comput. Fluids 22 (1993) 215.

[13] A. Dedner, F. Kemm, D. Kröner, C.D. Munz, T. Schnitzer and M. Wesenberg, Hyperbolic divergence cleaning for the MHD equations. J. Comput. Phys. 175 (2002) 645-673. 
[14] C. Evans and J.F. Hawley, Simulation of magnetohydrodynamic flow: a constrained transport method. Astrophys. J. 332 (1998) 659.

[15] M. Fey, Multi-dimensional upwingding.(I) The method of transport for solving the Euler equations. J. Comput. Phys. 143 (1998) 159-180.

[16] M. Fey, Multi-dimensional upwingding.(II) Decomposition of Euler equations into advection equations. J. Comput. Phys. 143 (1998) 181-199.

[17] F. Fuchs, S. Mishra and N.H. Risebro, Splitting based finite volume schemes for ideal MHD equations. J. Comput. Phys. 228 (2009) 641-660.

[18] F. Fuchs, A. McMurry, S. Mishra, N.H. Risebro and K. Waagan, Finite volume methods for wave propagation in stratified magneto-atmospheres. Commun. Comput. Phys. 7 (2010) 473-509.

[19] F. Fuchs, A.D. McMurry, S. Mishra, N.H. Risebro and K. Waagan, Approximate Riemann solver and robust high-order finite volume schemes for the MHD equations in multi-dimensions. Commun. Comput. Phys. 9 (2011) 324-362.

[20] S. Gottlieb, C.W. Shu and E. Tadmor, High order time discretizations with strong stability property. SIAM. Rev. 43 (2001) 89-112.

[21] K.F. Gurski, An HLLC-type approximate Riemann solver for ideal Magneto-hydro dynamics. SIAM. J. Sci. Comput. 25 (2004) 2165-2187.

[22] A. Harten, B. Engquist, S. Osher and S.R. Chakravarty, Uniformly high order accurate essentially non-oscillatory schemes. J. Comput. Phys. 71 (1987) 231-303.

[23] A. Kurganov and E. Tadmor, New high resolution central schemes for non-linear conservation laws and convection-diffusion equations. J. Comput. Phys. 160 (2000) 241-282.

[24] R.J. LeVeque, Wave propagation algorithms for multi-dimensional hyperbolic systems, J. Comput. Phys. 131 (1997) $327-353$.

[25] R.J. LeVeque, Finite volume methods for hyperbolic problems. Cambridge university press, Cambridge (2002).

[26] T.J. Linde, A three adaptive multi fluid MHD model for the heliosphere. Ph.D. thesis, University of Michigan, Ann-Arbor (1998).

[27] M. Lukacova-Medvidova, K.W. Morton and G. Warnecke, Evolution Galerkin methods for Hyperbolic systems in two space dimensions. Math. Comput. 69 (2000) 1355-1384.

[28] M. Lukacova-Medvidova, J. Saibertova and G. Warnecke, Finite volume evolution Galerkin methods for Non-linear hyperbolic systems. J. Comput. Phys. 183 (2003) 533-562.

[29] S. Mishra and E. Tadmor, Constraint preserving schemes using potential-based fluxes. I. Multi-dimensional transport equations. Commun. Comput. Phys. 9 (2010) 688-710.

[30] S. Mishra and E. Tadmor, Constraint preserving schemes using potential-based fluxes. II. Genuinely multi-dimensional systems of conservation laws. SIAM J. Numer. Anal. 49 (2011) 1023-1045.

[31] A. Mignone et al., Pluto: A numerical code for computational astrophysics. Astrophys. J. Suppl. 170 (2007) $228-242$.

[32] T. Miyoshi and K. Kusano, A multi-state HLL approximate Riemann solver for ideal magneto hydro dynamics. J. Comput. Phys. 208 (2005) 315-344.

[33] H. Nessyahu and E. Tadmor, Non-oscillatory central differencing for hyperbolic conservation laws. J. Comput. Phys. 87 (1990) 408-463.

[34] S. Noelle, The MOT-ICE: A new high-resolution wave propagation algorithm for multi-dimensional systems of conservation laws based on Fey's method of transport. J. Comput. Phys. 164 (2000) 283-334.

[35] K.G. Powell, An approximate Riemann solver for magneto-hydro dynamics (that works in more than one space dimension). Technical report, ICASE, Langley, VA (1994) 94-24.

[36] K.G. Powell, P.L. Roe, T.J. Linde, T.I. Gombosi and D.L. De zeeuw, A solution adaptive upwind scheme for ideal MHD. J. Comput. Phys. 154 (1999) 284-309.

[37] P.L. Roe and D.S. Balsara, Notes on the eigensystem of magnetohydrodynamics. SIAM. J. Appl. Math. 56 (1996) 57-67.

[38] J. Rossmanith, A wave propagation method with constrained transport for shallow water and ideal magnetohydrodynamics. Ph.D. thesis, University of Washington, Seattle (2002).

[39] D.S. Ryu, F. Miniati, T.W. Jones and A. Frank, A divergence free upwind code for multidimensional magnetohydrodynamic flows. Astrophys. J. 509 (1998) 244-255.

[40] C.W. Shu and S. Osher, Efficient implementation of essentially non-oscillatory schemes - II. J. Comput. Phys. 83 (1989) $32-78$.

[41] E. Tadmor, Approximate solutions of nonlinear conservation laws, in Advanced Numerical approximations of Nonlinear Hyperbolic equations, edited by A. Quarteroni. Lecture notes in Mathematics, Springer Verlag (1998) 1-149.

[42] M. Torrilhon, Locally divergence preserving upwind finite volume schemes for magnetohyrodynamic equations. SIAM. J. Sci. Comput. 26 (2005) 1166-1191.

[43] M. Torrilhon and M. Fey, Constraint-preserving upwind methods for multidimensional advection equations. SIAM. J. Numer. Anal. 42 (2004) 1694-1728.

[44] G. Toth, The $\operatorname{Div} B=0$ constraint in shock capturing magnetohydrodynamics codes. J. Comput. Phys. 161 (2000) 605-652.

[45] B. van Leer, Towards the ultimate conservative difference scheme, V. A second order sequel to Godunov's method. J. Comput. Phys. 32 (1979) 101-136. 